\title{
PT-symmetry breaking hamiltonian interactions in BF models
}

\author{
E. M. Cioroianu*, S. C. Săraru ${ }^{\dagger}$ \\ Faculty of Physics, University of Craiova \\ 13 A. I. Cuza Str., Craiova, 200585, Romania
}

\begin{abstract}
The PT-symmetry breaking, consistent hamiltonian interactions in all $n \geq 4$ spacetime dimensions that can be added to an abelian BF model involving a set of scalar fields, two sorts of one-forms, and a system of two-forms are obtained by means of the hamiltonian deformation procedure based on local BRST cohomology. This paper enhances one of our previous works, where only PT-invariant deformations were considered. The associated coupled theory is an interacting, topological BF model exhibiting an open gauge algebra and on-shell reducibility relations.

PACS number: 11.10.Ef
\end{abstract}

\section{Introduction}

The great advantage of the hamiltonian BRST symmetry [1, 2] is represented by its proper implementation in quantum mechanics [1] (Chapter 14), and also by an appropriate correlation with the canonical quantization methods [3. The understanding of this symmetry from a cohomological point of view made possible a unitary approach to many problems in gauge field theory, such as the hamiltonian analysis of anomalies [4, the precise relation between local lagrangian and hamiltonian BRST cohomologies [5], and, recently, the problem of obtaining consistent hamiltonian interactions in gauge theories by means of the deformation theory [6, 7, 8, 9, 10].

In this paper we investigate the PT-symmetry breaking, consistent hamiltonian deformations in any spacetime dimension $n \geq 4$ of a free abelian topological field theory of BF-type [1] involving a set of scalar fields, two collections of one-forms, and a system of two-forms. Actually, this work enhances our previous results from [8], where the interactions were imposed to preserve PT invariance. Here, we relax this condition and show that the resulting interactions are accurately described by a topological field theory with an open algebra of first-class constraints, that can be interpreted in terms of a Poisson structure present in various models of two-dimensional gravity [12, 13, 14, 15. [The analysis of Poisson Sigma Models, including their relationship to two-dimensional gravity and the study of classical solutions, can be found in [16, 17, 18, 19, 20, 21] (see also [22])].

The plan of the paper is the following. Section 2 briefly reviews the problem of constructing consistent hamiltonian interactions in the framework of the BRST formalism, which reduces to solving two towers of equations that describe the deformation of the BRST charge, respectively, of the BRST-invariant Hamiltonian associated with a given "free" first-class theory at various orders in the coupling constant. In Section 3 we determine the hamiltonian BRST symmetry $(s)$ of the free topological theory under study in $n \geq 4$ spacetime dimensions, which splits as the sum between the Koszul-Tate differential and the exterior derivative along the gauge orbits. This model is abelian and $(n-2)$-stage reducible, the reducibility relations holding off-shell (everywhere in the phase space). Next, we solve the main equations governing the hamiltonian deformation procedure

*e-mail address: manache@central.ucv.ro

†e-mail address: scsararu@central.ucv.ro 
on behalf of the BRST cohomology of the free theory. In Section 4 we initially compute, using specific cohomological techniques, the first-order deformation of the BRST charge, which lies in the cohomological space of $s$ modulo the spatial part of the exterior spacetime derivative $(\tilde{d})$ in ghost number one, $H^{1}(s \mid \tilde{d})$. The first-order deformation of the BRST charge stops at antighost number $(n-1)$ and contains two types of solutions: one that preserves the PT invariance and is described by two sorts of arbitrary functions involving only the undifferentiated scalar fields, previously investigated in [8], and the other that breaks the PT invariance and has not been considered in the literature so far. The latter deformation is parametrized by a completely antisymmetric 'tensor' of rank $n$ that involves only the undifferentiated scalar fields. The consistency of the first-order deformation imposes certain restrictions on these three types of functions depending only on the undifferentiated scalar fields and allows them to be parametrized in terms of a single 'two-tensor' (in the collection indices) depending on the scalar fields, that must be antisymmetric and fulfills a certain identity. The other two functions are obtained from the derivatives of this 'two-tensor' with respect to the scalar fields. Under these conditions, all the other deformations, of order two and higher, can be taken to vanish, and thus the BRST charge of the interacting model that is consistent to all orders in the deformation parameter is fully output. Section 5 solves the problem of generating the deformed BRST-invariant Hamiltonian, which can be taken nonzero only at the first order in the coupling constant. With the help of these deformed hamiltonian BRST quantities, in Section 6 we identify the interacting gauge theory, which is again topological and displays an open algebra of constraints (the Dirac brackets among the deformed first-class constraint functions only close on the first-class constraint surface). The deformed first-class constraints are of course reducible, but the reducibility relations hold on-shell (on the first-class constraint surface). It is interesting to observe that the relaxation of the condition on the PT invariance of the deformations brings in new, consistent, nontrivial terms at the level of both BRST charge and BRST-invariant Hamiltonian. The only sector that 'does not feel' the relaxation of this condition is the redundancy of the deformed first-class constraints, including both the reducibility relations and functions. Section 7 contains the main conclusions of the present paper. Two appendix sections complete the description of the interacting model.

\section{Main equations of the hamiltonian deformation procedure}

It has been shown in [6] that the problem of constructing consistent hamiltonian interactions in theories with first-class constraints can be equivalently reformulated as a deformation problem of the BRST charge $\Omega_{0}$ and of the BRST-invariant Hamiltonian $H_{0 \mathrm{~B}}$ of a given "free" first-class theory. More precisely, if the interactions can be consistently constructed, then the "free" BRST charge can be deformed into

$$
\begin{aligned}
\Omega_{0} \rightarrow \hat{\Omega} & =\Omega_{0}+g \int d^{n-1} x \hat{\omega}_{1}+g^{2} \int d^{n-1} x \hat{\omega}_{2}+O\left(g^{3}\right)= \\
& =\Omega_{0}+g \hat{\Omega}_{1}+g^{2} \hat{\Omega}_{2}+O\left(g^{3}\right)
\end{aligned}
$$

where the BRST charge of the interacting theory $\hat{\Omega}$ must satisfy the equation

$$
[\hat{\Omega}, \hat{\Omega}]=0 .
$$

The last relation projected on various powers in the deformation parameter $g$ is equivalent with the tower of equations

$$
\begin{aligned}
{\left[\Omega_{0}, \Omega_{0}\right] } & =0, \\
2\left[\Omega_{0}, \hat{\Omega}_{1}\right] & =0,
\end{aligned}
$$




$$
2\left[\Omega_{0}, \hat{\Omega}_{2}\right]+\left[\hat{\Omega}_{1}, \hat{\Omega}_{1}\right]=0
$$

In a similar manner the BRST-invariant Hamiltonian of the "free" theory can be deformed like

$$
\begin{aligned}
H_{0 \mathrm{~B}} \rightarrow \hat{H}_{\mathrm{B}} & =H_{0 \mathrm{~B}}+g \int d^{n-1} x \hat{h}_{1}+g^{2} \int d^{n-1} x \hat{h}_{2}+O\left(g^{3}\right)= \\
& =H_{0 \mathrm{~B}}+g \hat{H}_{1}+g^{2} \hat{H}_{2}+O\left(g^{3}\right),
\end{aligned}
$$

and it stands for the BRST-invariant Hamiltonian of the coupled system

$$
\left[\hat{H}_{\mathrm{B}}, \hat{\Omega}\right]=0 \text {. }
$$

The decomposition of the relation (7) according to the various orders in the coupling constant reveals a new tower of equations

$$
\begin{aligned}
{\left[H_{0 \mathrm{~B}}, \Omega_{0}\right] } & =0, \\
{\left[\hat{H}_{1}, \Omega_{0}\right]+\left[H_{0 \mathrm{~B}}, \hat{\Omega}_{1}\right] } & =0, \\
{\left[\hat{H}_{2}, \Omega_{0}\right]+\left[\hat{H}_{1}, \hat{\Omega}_{1}\right]+\left[H_{0 \mathrm{~B}}, \hat{\Omega}_{2}\right] } & =0,
\end{aligned}
$$

While, the equations (3) and (8) are satisfied since $\Omega_{0}$ and $H_{0 \mathrm{~B}}$ are by hypothesis the BRST charge and respectively the BRST-invariant Hamiltonian of the "free" theory, the resolution of the remaining equations (44)-(5), etc., and (90)-(10), etc.) by means of cohomological techniques provides the hamiltonian BRST description of the interacting gauge theory corresponding to the initial "free" one.

\section{$3 \quad$ Free BRST symmetry}

Our starting point is a free, topological field theory of BF-type in $n \geq 4$ spacetime dimensions that involves two types of one-forms, a collection of scalar fields, and a system of two-forms, described by the lagrangian action

$$
S_{0}\left[A_{\mu}^{a}, H_{\mu}^{a}, \varphi_{a}, B_{a}^{\mu \nu}\right]=\int d^{n} x\left(H_{\mu}^{a} \partial^{\mu} \varphi_{a}+\frac{1}{2} B_{a}^{\mu \nu} \partial_{[\mu} A_{\nu]}^{a}\right)
$$

where here and in the sequel the notation $[\mu \ldots \nu]$ (or $[i \ldots j]$ ) signifies full antisymmetry with respect to the indices between brackets without normalization factors (i.e. the independent terms appear only once and are not multiplied by overall numerical factors). The above action is invariant under the gauge transformations

$$
\delta_{\epsilon} A_{\mu}^{a}=\partial_{\mu} \epsilon^{a}, \quad \delta_{\epsilon} H_{\mu}^{a}=\partial^{\nu} \epsilon_{\mu \nu}^{a}, \quad \delta_{\epsilon} \varphi_{a}=0, \quad \delta_{\epsilon} B_{a}^{\mu \nu}=\partial_{\rho} \epsilon_{a}^{\mu \nu \rho},
$$

which are off-shell $(n-2)$-stage reducible, where the gauge parameters $\epsilon^{a}, \epsilon_{\mu \nu}^{a}$, and $\epsilon_{a}^{\mu \nu \rho}$ are bosonic, the last two sets being completely antisymmetric.

After the elimination of the second-class constraints (the coordinates of the reduced phase-space are $\left.z^{A}=\left(\pi_{a}^{0}, A_{\mu}^{a}, B_{a}^{\mu \nu}, p_{a}^{i}, H_{\mu}^{a}, \pi_{i j}^{a}, \varphi_{a}\right)\right)$, we are left with a system subject only to the first-class constraints

$$
\begin{aligned}
G_{a}^{(1)} & \equiv \pi_{a}^{0} \approx 0, \quad G_{a}^{(2)} \equiv-\partial_{i} B_{a}^{0 i} \approx 0, \\
G_{i j}^{(1) a} & \equiv 2 \pi_{i j}^{a} \approx 0, \quad G_{i j}^{(2) a} \equiv-\partial_{[i} A_{j]}^{a} \approx 0, \\
\gamma_{a}^{(1) i} & \equiv-p_{a}^{i} \approx 0, \quad \gamma_{a}^{(2) i} \equiv \partial^{i} \varphi_{a} \approx 0,
\end{aligned}
$$


and displaying the first-class Hamiltonian

$$
H_{0}=\int d^{n-1} x\left(-H_{i}^{a} \gamma_{a}^{(2) i}+\frac{1}{2} B_{a}^{i j} G_{i j}^{(2) a}+A_{0}^{a} G_{a}^{(2)}\right),
$$

in terms of the non-vanishing fundamental Dirac brackets

$$
\begin{aligned}
{\left[\pi_{a}^{0}(t, \mathbf{x}), A_{0}^{b}(t, \mathbf{y})\right] } & =-\delta_{a}^{b} \delta^{n-1}(\mathbf{x}-\mathbf{y}) \\
{\left[B_{a}^{0 i}(t, \mathbf{x}), A_{j}^{b}(t, \mathbf{y})\right] } & =-\delta_{j}^{i} \delta_{a}^{b} \delta^{n-1}(\mathbf{x}-\mathbf{y}), \\
{\left[H_{0}^{a}(t, \mathbf{x}), \varphi_{b}(t, \mathbf{y})\right] } & =-\delta_{b}^{a} \delta^{n-1}(\mathbf{x}-\mathbf{y}), \\
{\left[\pi_{i j}^{a}(t, \mathbf{x}), B_{b}^{k l}(t, \mathbf{y})\right] } & =-\frac{1}{2} \delta_{i}^{[k} \delta_{j}^{l]} \delta_{b}^{a} \delta^{n-1}(\mathbf{x}-\mathbf{y}), \\
{\left[p_{a}^{i}(t, \mathbf{x}), H_{j}^{b}(t, \mathbf{y})\right] } & =-\delta_{j}^{i} \delta_{a}^{b} \delta^{n-1}(\mathbf{x}-\mathbf{y}) .
\end{aligned}
$$

The above constraints are abelian, while the remaining gauge algebra relations are expressed by

$$
\begin{aligned}
{\left[H_{0}, G_{a}^{(1)}\right] } & =G_{a}^{(2)}, \quad\left[H_{0}, G_{a}^{(2)}\right]=0 \\
{\left[H_{0}, G_{i j}^{(1) a}\right] } & =G_{i j}^{(2) a}, \quad\left[H_{0}, G_{i j}^{(2) a}\right]=0 \\
{\left[H_{0}, \gamma_{a}^{(1) i}\right] } & =\gamma_{a}^{(2) i}, \quad\left[H_{0}, \gamma_{a}^{(2) i}\right]=0 .
\end{aligned}
$$

The constraint functions $G_{i j}^{(2) a}$ are off-shell $(n-3)$-stage reducible, with the reducibility functions (of order $(k-2))$ given by

$$
\left(Z_{i_{1} i_{2} \ldots i_{k}}^{a}\right)_{b}^{j_{1} \ldots j_{k-1}}=\frac{(-)^{k-1}}{(k-1) !} \delta_{b}^{a} \partial_{\left[i_{1}\right.} \delta_{i_{2}}^{j_{1}} \cdots \delta_{\left.i_{k}\right]}^{j_{k-1}}, \quad k=\overline{3, n-1},
$$

while the constraint functions $\gamma_{a}^{(2) i}$ are off-shell $(n-2)$-stage reducible, the associated reducibility functions (of order $(k-1)$ ) being

$$
\left(Z_{a}^{i_{1} i_{2} \ldots i_{k}}\right)_{j_{1} \ldots j_{k-1}}^{b}=\frac{(-)^{k-1}}{(k-1) !} \delta_{a}^{b} \partial^{\left[i_{1}\right.} \delta_{j_{1}}^{i_{2}} \cdots \delta_{j_{k-1}}^{\left.i_{k}\right]}, \quad k=\overline{2, n-1}
$$

The hamiltonian BRST formalism requires the introduction of the ghosts

$$
\begin{aligned}
\eta^{a_{0}} & =\left(\eta^{(1) a}, \eta^{a}, \eta_{a}^{(1) i j}, \eta_{a}^{i j}, C_{i}^{(1) a}, C_{i}^{a}\right), \\
\eta^{a_{k}} & =\left(C_{i_{1} \ldots i_{k+1}}^{a}, \eta_{a}^{i_{1} \ldots i_{k+2}}\right), \quad k=\overline{1, n-3} \\
\eta^{a_{n-2}} & =\left(C_{i_{1} \ldots i_{n-1}}^{a}\right),
\end{aligned}
$$

together with their conjugated antighosts

$$
\begin{aligned}
\mathcal{P}_{a_{0}} & =\left(\mathcal{P}_{a}^{(1)}, \mathcal{P}_{a}, \mathcal{P}_{i j}^{(1) a}, \mathcal{P}_{i j}^{a}, P_{a}^{(1) i}, P_{a}^{i}\right), \\
\mathcal{P}_{a_{k}} & =\left(P_{a}^{i_{1} \ldots i_{k+1}}, \mathcal{P}_{i_{1} \ldots i_{k+2}}^{a}\right), \quad k=\overline{1, n-3}, \\
\mathcal{P}_{a_{n-2}} & =\left(P_{a}^{i_{1} i_{2} \ldots i_{n-1}}\right) .
\end{aligned}
$$

The first set of ghosts respectively corresponds to the first-class constraints (13)-(15), while the other two are due to the reducibility of the first-class constraint functions. The fields $\eta^{a_{0}}$ in (27) are fermionic, the fields $\eta^{a_{k}}$ in (28) possess the Grassmann parity $(k+1) \bmod 2$, while those in (29) have the Grassmann parity $(n-1) \bmod 2$. The ghost number and Grassmann parity of the 
antighosts follow from the general rules of the standard hamiltonian BRST formalism. The ghost number is defined in usual manner as the difference between the pure ghost number (pgh) and the antighost number (agh), where

$$
\begin{aligned}
\operatorname{pgh}\left(z^{A}\right) & =0, \operatorname{pgh}\left(\eta^{a_{0}}\right)=1, \quad \operatorname{pgh}\left(\mathcal{P}_{a_{0}}\right)=0, \\
\operatorname{pgh}\left(\eta^{a_{k}}\right) & =k+1, \quad \operatorname{pgh}\left(\mathcal{P}_{a_{k}}\right)=0, \quad k=\overline{1, n-3}, \\
\operatorname{pgh}\left(\eta^{a_{n-2}}\right) & =n-1, \quad \operatorname{pgh}\left(\mathcal{P}_{a_{n-2}}\right)=0, \\
\operatorname{agh}\left(z^{A}\right) & =0, \quad \operatorname{agh}\left(\eta^{a_{0}}\right)=0, \quad \operatorname{agh}\left(\mathcal{P}_{a_{0}}\right)=1, \\
\operatorname{agh}\left(\eta^{a_{k}}\right) & =0, \quad \operatorname{agh}\left(\mathcal{P}_{a_{k}}\right)=k+1, \quad k=\overline{1, n-3}, \\
\operatorname{agh}\left(\eta^{a_{n-2}}\right) & =0, \quad \operatorname{agh}\left(\mathcal{P}_{a_{n-2}}\right)=n-1 .
\end{aligned}
$$

The BRST charge of this free model takes the form

$$
\begin{aligned}
& \Omega_{0}=\int d^{n-1} x\left(\eta^{(1) a} G_{a}^{(1)}+\eta^{a} G_{a}^{(2)}+\eta_{a}^{(1) i j} G_{i j}^{(1) a}+\eta_{a}^{i j} G_{i j}^{(2) a}+C_{i}^{(1) a} \gamma_{a}^{(1) i}\right. \\
& \left.+C_{i}^{a} \gamma_{a}^{(2) i}+\sum_{k=3}^{n-1}(-)^{k-1} \eta_{a}^{i_{1} i_{2} \ldots i_{k}} \partial_{\left[i_{1}\right.} \mathcal{P}_{\left.i_{2} \ldots i_{k}\right]}^{a}+\sum_{k=2}^{n-1}(-)^{k-1} C_{i_{1} i_{2} \ldots i_{k}}^{a} \partial^{\left[i_{1}\right.} P_{a}^{\left.i_{2} \ldots i_{k}\right]}\right)
\end{aligned}
$$

while the corresponding BRST-invariant Hamiltonian is expressed like

$$
H_{0 \mathrm{~B}}=H_{0}+\int d^{n-1} x\left(\eta^{(1) a} \mathcal{P}_{a}+\eta_{a}^{(1) i j} \mathcal{P}_{i j}^{a}+C_{i}^{(1) a} P_{a}^{i}\right)
$$

In general, any function $F$ with $\operatorname{gh}(F)=0$ that is BRST-closed, $\left[F, \Omega_{0}\right]=0$, is called BRST observables. Due to the topological behavior of this model (the number of physical degrees of freedom is equal to zero), all the BRST observables are trivial (BRST-exact), meaning that each of them can be written like $F=\left[M_{0}, \Omega_{0}\right]$, for some fermionic $M_{0}$ with gh $\left(M_{0}\right)=-1$. In particular, the BRST-invariant Hamiltonian is BRST-exact

$$
H_{0 \mathrm{~B}}=\left[K_{0}, \Omega_{0}\right]
$$

where, in this situation,

$$
K_{0}=\int d^{n-1} x\left(H_{i}^{a} P_{a}^{i}-\frac{1}{2} B_{a}^{i j} \mathcal{P}_{i j}^{a}-A_{0}^{a} \mathcal{P}_{a}\right)
$$

The BRST symmetry of the free theory, $s \cdot=\left[\cdot, \Omega_{0}\right]$, splits as

$$
s=\delta+\gamma,
$$

where $\delta$ denotes the Koszul-Tate differential $(\operatorname{agh}(\delta)=-1, \operatorname{pgh}(\delta)=0)$, and $\gamma$ represents the exterior longitudinal derivative $(\operatorname{agh}(\gamma)=0, \operatorname{pgh}(\gamma)=1)$. These two operators act on the variables from BRST complex like

$$
\begin{gathered}
\delta z^{A}=0, \quad \delta \eta^{a_{k}}=0, \quad k=\overline{0, n-2} \\
\delta \mathcal{P}_{a}^{(1)}=-\pi_{a}^{0}, \quad \delta \mathcal{P}_{a}=\partial_{i} B_{a}^{0 i}, \quad \delta P_{a}^{(1) i}=p_{a}^{i}, \quad \delta P_{a}^{i}=-\partial^{i} \varphi_{a}, \\
\delta \mathcal{P}_{i j}^{(1) a}=-2 \pi_{i j}^{a}, \quad \delta \mathcal{P}_{i j}^{a}=\partial_{[i} A_{j]}^{a}, \\
\delta P_{a}^{i_{1} i_{2} \ldots i_{k}}=(-)^{k} \partial^{\left[i_{1}\right.} P_{a}^{\left.i_{2} \ldots i_{k}\right]}, \quad k=\overline{2, n-1} \\
\delta \mathcal{P}_{i_{1} i_{2} \ldots i_{k}}^{a}=(-)^{k} \partial_{\left[i_{1}\right.} \mathcal{P}_{\left.i_{2} \ldots i_{k}\right]}^{a}, \quad k=\overline{3, n-1}, \\
\gamma A_{i}^{a}=\partial_{i} \eta^{a}, \quad \gamma A_{0}^{a}=\eta^{(1) a}, \quad \gamma \varphi_{a}=0, \quad \gamma \pi_{a}^{0}=0, \quad \gamma p_{a}^{i}=0, \quad \gamma \pi_{i j}^{a}=0
\end{gathered}
$$




$$
\begin{gathered}
\gamma B_{a}^{0 i}=2 \partial_{j} \eta_{a}^{i j}, \quad \gamma B_{a}^{i j}=2 \eta_{a}^{(1) i j}, \quad \gamma H_{i}^{a}=-C_{i}^{(1) a}, \quad \gamma H_{0}^{a}=\partial^{i} C_{i}^{a} \\
\gamma \eta^{(1) a}=\gamma \eta^{a}=\gamma C_{i}^{(1) a}=\gamma \eta_{a}^{(1) i j}=0 \\
\gamma \eta_{a}^{i j}=3 \partial_{k} \eta_{a}^{i j k}, \quad \gamma C_{i}^{a}=2 \partial^{j} C_{i j}^{a}, \\
\gamma \eta_{a}^{i_{1} \ldots i_{k}}=(k+1) \partial_{i} \eta_{a}^{i i_{1} \ldots i_{k}}, \quad k=\overline{3, n-2} \\
\gamma C_{i_{1} \ldots i_{k}}^{a}=-(k+1) \partial^{i} C_{i i_{1} \ldots i_{k}}^{a}, \quad k=\overline{2, n-2} \\
\gamma \eta_{a}^{i_{1} \ldots i_{n-1}}=0, \quad \gamma C_{i_{1} \ldots i_{n-1}}^{a}=0 \\
\gamma \mathcal{P}_{a_{k}}=0, \quad k=\overline{0, n-2} .
\end{gathered}
$$

The last formulas will be employed in the next section at the deformation of the free theory.

\section{Deformation of the BRST charge}

In this section we solve the equations (4) -(5), etc., that govern the deformation of the BRST charge in the case of the topological free model under study by relying on cohomological techniques. As a result, we find that only the first-order deformation is nontrivial, while its consistency is equivalent to the existence of a Poisson 'two-tensor' (in the collection indices) depending on the undifferentiated scalar fields, that must be antisymmetric and fulfills a certain identity. This twotensor, together with its derivatives with respect to the scalar fields, parametrizes the final form of the BRST charge. Two main types of deformations of the BRST charge are considered: one that breaks the PT invariance and the other that preserves it. Although unrelated at the level of the first-order deformation, these two kinds of solutions become connected when passing to the higherorder deformations. More precisely, the part that breaks the PT invariance is initially parametrized by some completely antisymmetric 'tensor' of rank $n$, where $n$ is the spacetime dimension, which involves only the undifferentiated scalar fields from the collection. However, the consistency of the first-order deformation requires that this antisymmetric 'tensor' is expressed precisely via the derivatives of the Poisson 'two-tensor' that parametrizes the PT-invariant solution.

\subsection{First-order deformation}

Initially, we solve the equation (41), which is responsible for the first-order deformation of the BRST charge. It takes the local form

$$
s \hat{\omega}_{1}=\partial_{i} \hat{j}^{i}
$$

for some local $\hat{j}^{i}$. In order to simplify the exposition, we represent $\hat{\omega}_{1}$ like

$$
\hat{\omega}_{1}=\omega_{1}+\bar{\omega}_{1}
$$

where $\omega_{1}$ is the component of the first-order deformation of the BRST charge that preserves the PT invariance and $\bar{\omega}_{1}$ is the piece that breaks the PT invariance. The concrete form of $\omega_{1}$ has been obtained in [8] and is briefly exposed in the Appendix A] As it has been shown in [8], $\omega_{1}$ satisfies individually an equation of the type (57), and therefore the decomposition (58) and the equation (57) require that $\bar{\omega}_{1}$ must separately verify a similar equation, i.e.

$$
s \bar{\omega}_{1}=\partial_{i} \bar{j}^{i} .
$$

In order to investigate the solutions to this equation, we develop $\bar{\omega}_{1}$ according to the antighost number and suppose that the development stops at a finite order

$$
\bar{\omega}_{1}=\stackrel{(0)}{\omega}_{1}+\stackrel{(1)}{\omega}_{1}+\cdots+\stackrel{(J)}{\omega}_{1}, \quad \operatorname{agh}\left(\stackrel{(I)}{\omega}_{1}\right)=I, \quad \operatorname{gh}\left(\stackrel{(I)}{\omega}_{1}\right)=1
$$


where the last term can be assumed to be annihilated by $\gamma$

$$
\gamma \stackrel{(J)}{\omega}_{1}=0
$$

Both results can be shown by adapting the standard lagrangian arguments from 23 to the hamiltonian formulation. Thus, we need to compute the cohomology of the exterior longitudinal derivative, $H(\gamma)$, in order to determine the piece of highest antighost number in (60).

With the help of the definitions (49)-(56) of $\gamma$ acting on the BRST generators, we remark that every local representative of $H(\gamma)$ is generated by

$$
\Phi^{\alpha}=\left(F_{i j}^{a}=\partial_{[i} A_{j]}^{a}, \varphi_{a}, \pi_{a}^{0}, p_{a}^{i}, \pi_{i j}^{a}, \partial_{i} B_{a}^{0 i}\right),
$$

(together with their spatial derivatives up to a finite order), by the antighosts (30)-(32) and their spatial derivatives up to a finite order, as well by the undifferentiated ghosts $\eta^{a}, \eta_{a}^{i_{1} \ldots i_{n-1}}$, and $C_{i_{1} \ldots i_{n-1}}^{a}$. (The ghosts $\eta^{(1) a}, C_{i}^{(1) a}$, and $\eta_{a}^{(1) i j}$, although $\gamma$-invariant, are also $\gamma$-exact, and hence trivial in $H(\gamma)$. The same is true with respect to the spatial part of the spacetime derivatives of $\eta^{a}, \eta_{a}^{i_{1} \ldots i_{n-1}}$, and $C_{i_{1} \ldots i_{n-1}}^{a}$.) In this way, the general, local solution to the equation (61) can be written (up to trivial, $\gamma$-exact contributions) as

$$
\stackrel{(J)}{\omega}_{1}=a_{J}\left(\left[\Phi^{\alpha}\right],\left[\mathcal{P}_{a_{k}}\right]_{k=\overline{0, n-2}}\right) e^{J+1}\left(\eta^{a}, \eta_{a}^{i_{1} \ldots i_{n-1}}, C_{i_{1} \ldots i_{n-1}}^{a}\right),
$$

where $e^{J+1}\left(\eta^{a}, \eta_{a}^{i_{1} \ldots i_{n-1}}, C_{i_{1} \ldots i_{n-1}}^{a}\right)$ stand for the elements with pure ghost number equal to $(J+1)$ of a basis in the space of the polynomials in the corresponding ghosts, and $a_{J}$ are $\gamma$-closed elements of pure ghost number zero, with bounded antighost number, agh $\left(a_{J}\right)=J$. The objects $a_{J}$ play here the role of 'invariant polynomials' 24] from the lagrangian approach. The notation $f([q])$ signifies that $f$ depends on $q$ and its spatial derivatives up to a finite order.

The equation (59) projected on antighost number $(J-1)$ becomes

$$
\delta \stackrel{(J)}{\bar{\omega}}_{1}+\gamma \stackrel{(J-1)}{\bar{\omega}}_{1}=\partial_{i} \stackrel{(J)}{m}^{i}
$$

Introducing (63) in (64), it follows that a necessary condition for the existence of (nontrivial) ${ }^{(J-1)}{ }_{1}$ is that the 'invariant polynomials' $a_{J}$ from (63) are (nontrivial) elements of $H_{J}(\delta \mid \tilde{d})$, where the last notation means the cohomological space of the Koszul-Tate differential modulo the spatial part of the exterior spacetime derivative in pure ghost number zero and in strictly positive antighost number $J$

$$
\delta a_{J}=\partial^{i} n_{i}, \quad \operatorname{agh}\left(n_{i}\right)=J-1, \quad \operatorname{pgh}\left(n_{i}\right)=0 .
$$

Translating the lagrangian results from 24] regarding the triviality of the characteristic cohomology for linear gauge theories at the hamiltonian level, since our model is $(n-2)$-order reducible and the constraint functions are linear in the reduced phase-space variables, we can state that

$$
H_{K}(\delta \mid \tilde{d})=0 \quad \text { for all } \quad K>n-1
$$

The natural question raises, namely, if the result (66) is still valid in the space of 'invariant polynomials' $H^{\text {inv }}(\delta \mid \tilde{d})$, where an element of $H_{J}^{\text {inv }}(\delta \mid \tilde{d})$ is defined like in (65), but with both $a_{J}$ and $n_{i}$ 'invariant polynomials'. By analyzing what happens in most gauge theories at the lagrangian level, it is quite reasonable to assume the validity of (66) in the space of 'invariant polynomials'

$$
H_{K}^{\mathrm{inv}}(\delta \mid \tilde{d})=0 \quad \text { for all } \quad K>n-1
$$


Moreover, (67) is a consequence of the more general result that if $a_{K}$ is an 'invariant polynomial' with $\operatorname{agh}\left(a_{K}\right)=K \geq n-1$, which is trivial in $H_{K}(\delta \mid \tilde{d}), a_{K}=\delta b_{K}+\partial^{i} m_{i}$, with $\operatorname{agh}\left(b_{K}\right)=K+1$ and $\operatorname{agh}\left(m_{i}\right)=K$, then it can be taken to be trivial also in $H_{K}^{\text {inv }}(\delta \mid \tilde{d})$, i.e., both $b_{K}$ and $m_{i}$ can be taken to be 'invariant polynomials'.

The previous results on $H(\delta \mid \tilde{d})$ and $H^{\text {inv }}(\delta \mid \tilde{d})$ in strictly positive antighost numbers are important because they control the obstructions to removing the antighosts from the first-order deformation of the BRST charge. More precisely, one can successively eliminate all the pieces of antighost number strictly greater than $(n-1)$ from $\bar{\omega}_{1}$ by adding only trivial terms, so one can take, without loss of nontrivial objects, the condition $J \leq n-1$ in the decomposition (60). Moreover, the last representative can always be taken to belong to $H(\gamma)$, with the corresponding 'invariant polynomial' a nontrivial object from $H_{J}^{\text {inv }}(\delta \mid \tilde{d})$ for $J>1$ and respectively from $H_{1}(\delta \mid \tilde{d})$ if $J=1$.

Consequently, we can assume that $J=n-1$ in (60)

$$
\bar{\omega}_{1}=\stackrel{(0)}{\bar{\omega}}_{1}+{\stackrel{(1)}{\bar{\omega}^{\prime}}}_{1}+\cdots+\stackrel{(n-1)}{\bar{\omega}}_{1},
$$

with $\stackrel{(n-1)}{\bar{\omega}}_{1}$ given by $\left(\underline{63)}\right.$ for $J=n-1$ and $a_{n-1}$ a nontrivial element from $H_{n-1}^{\text {inv }}(\delta \mid \tilde{d})$. After some computation, we find that the most general representative of both $H_{n-1}(\delta \mid \tilde{d})$ and $H_{n-1}^{\text {inv }}(\delta \mid \tilde{d})$ can be expressed like

$$
\begin{aligned}
& a_{n-1}^{i_{1} \ldots i_{n-1}}=\frac{\partial U}{\partial \varphi_{a}} P_{a}^{i_{1} \ldots i_{n-1}}+\sum_{p=2}^{n-1} \sum_{1 \leq j_{1} \leq j_{2} \leq \cdots \leq j_{p}<n-1} \frac{\partial^{p} U}{\partial \varphi_{a_{1}} \partial \varphi_{a_{2}} \cdots \partial \varphi_{a_{p}}} \times \\
& \times P_{a_{1}}^{\left[i_{1} \ldots i_{j_{1}}\right.} P_{a_{2}}^{i_{j_{1}+1} \ldots i_{j_{1}+j_{2}}} \cdots P_{a_{p-1}}^{i_{j_{1}+\cdots+j_{p-2}+1} \ldots i_{j_{1}+\cdots+j_{p-1}}} P_{a_{p}}^{\left.i_{j_{1}+\cdots+j_{p-1}+1} \ldots i_{n-1}\right]},
\end{aligned}
$$

where $U$ is an arbitrary function involving only the undifferentiated scalar fields $\varphi_{a}$, and $j_{p}$ means

$$
j_{p}=n-1-\left(j_{1}+j_{2}+\cdots+j_{p-1}\right) .
$$

Taking into account the definitions (44)-(48) of the Koszul-Tate differential, one can prove the recursive relations

$$
\delta a_{k}^{i_{1} i_{2} \ldots i_{k}}=(-)^{k} \partial^{\left[i_{1}\right.} a_{k-1}^{\left.i_{2} \ldots i_{k}\right]}, \quad k=\overline{1, n-1},
$$

where for $k=\overline{2, n-2}$ we have

$$
\begin{aligned}
& a_{k}^{i_{1} \ldots i_{k}}=\frac{\partial U}{\partial \varphi_{a}} P_{a}^{i_{1} \ldots i_{k}}+\sum_{q=2}^{k} \sum_{1 \leq j_{1} \leq j_{2} \leq \cdots \leq j_{q}<k} \frac{\partial^{q} U}{\partial \varphi_{a_{1}} \partial \varphi_{a_{2}} \cdots \partial \varphi_{a_{q}}} \times \\
& \times P_{a_{1}}^{\left[i_{1} \ldots i_{j_{1}}\right.} P_{a_{2}}^{i_{j_{1}+1} \ldots i_{j_{1}+j_{2}}} \cdots P_{a_{q-1}}^{i_{j_{1}+\cdots+j_{q-2}+1} \ldots i_{j_{1}+\cdots+j_{q-1}}} P_{a_{q}}^{\left.i_{j_{1}+\cdots+j_{q-1}+1} \ldots i_{k}\right]},
\end{aligned}
$$

while for $k=1$ and respectively $k=0$ we obtain

$$
a_{1}^{i}=\frac{\partial U}{\partial \varphi_{a}} P_{a}^{i}, \quad a_{0}=U
$$

In (72) we used the notation $j_{q}=k-\left(j_{1}+j_{2}+\cdots+j_{q-1}\right)$. Now, we can completely determine the last component in (68). The elements of pure ghost number equal to $n, e^{n}\left(\eta^{a}, \eta_{a}^{i_{1} \ldots i_{n-1}}, C_{i_{1} \ldots i_{n-1}}^{a}\right)$, are given by

$$
e^{n}:\left(\eta^{a} C_{i_{1} \ldots i_{n-1}}^{b}, \eta^{a} \eta^{b} \eta_{c}^{i_{1} \ldots i_{n-1}}, \eta^{a_{1}} \eta^{a_{2}} \cdots \eta^{a_{n}}\right)
$$


for all $n \geq 4^{1}$. It means that the piece of highest antighost number in the first-order deformation is fully determined once we 'glue' (69) to (74) like in (63). The last component of $e^{n}$ needs the adjustment of a completely antisymmetric constant $K_{i_{1} \ldots i_{n-1}}$ in order to match (69), which can only be, by 'covariance' arguments, proportional to the spatial part of the completely antisymmetric symbol in $n$ dimensions, $\varepsilon_{0 i_{1} \ldots i_{n-1}}$. Even if we 'force' the introduction of additional antisymmetric symbols in the components of $\hat{\omega}_{1}$ involving the first two elements in (174), we finally obtain that such terms are always proportional with some objects that contain no antisymmetric symbols, like in (132). In conclusion, there is no possibility to construct pieces from $\stackrel{(n-1)}{\omega}_{1}$ that involve either of the first two elements in (74). Such terms can only bring contributions to the element $\stackrel{(n-1)}{\omega}_{1}$ of highest antighost number in the first-order deformation of the BRST charge that preserves the PT invariance, $\omega_{1}$.

As we have stated in the beginning of this section, here we focus only on the interactions that break the PT invariance and which, by virtue of the above discussion, can be generated just by the third element in (74), such that we can write

$$
\stackrel{(n-1)}{\bar{\omega}}_{1}=\frac{(-)^{\left[\frac{n}{2}\right]}}{n !} \epsilon_{0 i_{1} \ldots i_{n-1}} N_{a_{1} \ldots a_{n}}^{i_{1} \ldots i_{n-1}} \eta^{a_{1}} \cdots \eta^{a_{n}}
$$

where $\left[\frac{n}{2}\right]$ denotes the integer part of $\frac{n}{2}$. The element $N_{a_{1} \ldots a_{n}}^{i_{1} \ldots i_{n-1}}$ in (75) results from $a_{n-1}^{i_{1} i_{2} \ldots i_{n-1}}$ in (69) where we replace the function $U$ depending only on the undifferentiated scalar fields with a completely antisymmetric 'tensor' of rank $n, N_{a_{1} \ldots a_{n}}$, also involving just the $\varphi_{a}$ 's

$$
\begin{aligned}
& N_{a_{1} \ldots a_{n}}^{i_{1} \ldots i_{n-1}}=\frac{\partial N_{a_{1} \ldots a_{n}}}{\partial \varphi_{b}} P_{b}^{i_{1} i_{2} \ldots i_{n-1}}+\sum_{p=2}^{n-1} \sum_{1 \leq j_{1} \leq j_{2} \leq \cdots \leq j_{p}<n-1} \frac{\partial^{p} N_{a_{1} \ldots a_{n}}}{\partial \varphi_{b_{1}} \partial \varphi_{b_{2}} \cdots \partial \varphi_{b_{p}}} \times \\
& \times P_{b_{1}}^{\left[i_{1} \ldots i_{j_{1}}\right.} P_{b_{2}}^{i_{j_{1}+1 \ldots i_{j_{1}+j_{2}}} \cdots P_{b_{p-1}}^{i_{j_{1}+\cdots+j_{p-2}+1} \ldots i_{j_{1}+\cdots+j_{p-1}}} P_{b_{p}}^{\left.i_{j_{1}+\cdots+j_{p-1}+1} \ldots i_{n-1}\right]} .}
\end{aligned}
$$

The supplementary numerical factor from (75) has been added for further convenience.

Introducing the relation (75) into the equation (64) for $J=n-1$ and using the definitions (44) - (56), we infer that the piece with the antighost number equal to $(n-2)$ from the first-order deformation of the BRST charge that breaks the PT invariance reads as

$$
\stackrel{(n-2)}{\bar{\omega}}{ }_{1}=\frac{(-)^{\left[\frac{n}{2}\right]+1}}{(n-1) !} \sum_{p \geq 1}(-)^{p} \epsilon_{0 i_{1} \ldots i_{n-1-p} \ldots i_{n-1}} N_{a_{1} \ldots a_{n}}^{\left[i_{1} \ldots i_{n-1-p}\right.} \mathcal{P}^{\left.a_{1} i_{n-p} \ldots i_{n-1}\right]} \eta^{a_{2}} \cdots \eta^{a_{n}} .
$$

If we take into account the decomposition (43) of the free BRST differential and insert the expansion (68) into the equation (59), it follows that the component $\stackrel{(n-3)}{\bar{\omega}}{ }_{1}$ is solution to the equation (64) with $J \rightarrow n-2$. Substituting the solution (77) into this equation and using the definitions (44)-(56), after some computation we find that

$$
\begin{aligned}
\stackrel{(n-3)}{\bar{\omega}}{ }_{1}= & \frac{(-)^{\left[\frac{n}{2}\right]+2}}{(n-2) !} \sum_{p_{1} \geq p_{2} \geq 1}(-)^{p_{1}} \epsilon_{0 i_{1} \ldots i_{n-1-\left(p_{1}+p_{2}\right) \ldots i_{n-1}} N_{a_{1} \ldots a_{n}}^{\left[i_{1} \ldots i_{n-1-\left(p_{1}+p_{2}\right)}\right.} \times} \\
& \times \mathcal{P}^{a_{1} i_{n-\left(p_{1}+p_{2}\right) \cdots i_{n-1-p_{2}}}} \mathcal{P}^{\left.a_{2} i_{n-p_{2}} \ldots i_{n-1}\right]} \eta^{a_{3}} \cdots \eta^{a_{n}} .
\end{aligned}
$$

\footnotetext{
${ }^{1}$ For $n=4$ there is an extra possibility because $\eta_{a}^{i_{1} \cdots i_{n-1}} \rightarrow \eta_{a}^{i j k}$, with $\operatorname{pgh}\left(\eta_{a}^{i j k}\right)=2$, and so we have a supplementary element of the basis in the ghosts at pure ghost number $n=4$, namely, $\eta_{a}^{i j k} \eta_{b}^{i^{\prime} j^{\prime} k^{\prime}}$. However, this element can be discarded [10, so finally (74) still covers all the investigated situations.
} 
In a similar manner we solve the equations that govern the terms of antighost number $(n-m)$ from $\bar{\omega}_{1}$ with $m=\overline{4, n-2}$, which are expressed by (64) with $J \rightarrow n-m+1$, and get that

$$
\begin{aligned}
& \stackrel{(n-m)}{\bar{\omega}}_{1}=\frac{(-)^{\left[\frac{n}{2}\right]+m-1}}{(n-m+1) !} \sum_{p_{1} \geq p_{2} \geq \cdots \geq p_{m-1} \geq 1}(-)^{\Xi} \epsilon_{0 i_{1} \ldots i_{n-1-\left(p_{1}+p_{2}+\cdots+p_{m-1}\right)} \ldots i_{n-1}} \times
\end{aligned}
$$

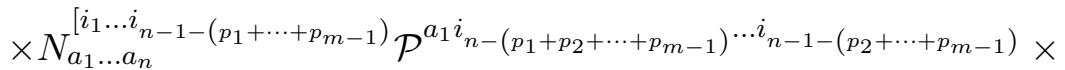

$$
\begin{aligned}
& \times \mathcal{P}^{\left.\left.a_{2} i_{n-\left(p_{2}+\cdots+p_{m-1}\right.}\right) \cdots i_{n-1-\left(p_{3}+\cdots+p_{m-1}\right.}\right)} \cdots \mathcal{P}^{\left.a_{m-1} i_{n-p_{m-1}} \cdots i_{n-1}\right]} \eta^{a_{m}} \cdots \eta^{a_{n}} \text {, }
\end{aligned}
$$

where we made the notations $M=\left(\left[\frac{m}{2}\right]-1\right)$ and $\Xi \equiv p_{1}+p_{3}+\cdots+p_{2 M+1}$. In the equations (77) - (79) we denoted by $\mathcal{P}^{a i}$ the spatial part of the collection of one-forms $A^{a \mu}$

$$
\mathcal{P}^{a i} \equiv A^{a i}
$$

in order to write the pieces that compose the first-order deformation of the BRST charge $\bar{\omega}_{1}$ in a brief, compact manner. We proceed in the same way with the equation (64) for $J \rightarrow 2$ and $J \rightarrow 1$, and determine the elements of antighost number one and respectively zero from $\bar{\omega}_{1}$ in the form

$$
\begin{aligned}
\stackrel{(1)}{\omega}_{1}= & \frac{1}{2} \epsilon_{0 i_{1} \ldots i_{n-1}}\left(-N_{a_{1} \ldots a_{n}}^{\left[i_{1}\right.} A^{a_{1} i_{2}} A^{a_{2} i_{3}} \cdots A^{\left.a_{n-2} i_{n-1}\right]}\right. \\
& \left.+N_{a_{1} \ldots a_{n}} \mathcal{P}^{a_{1}\left[i_{1} i_{2}\right.} A^{a_{2} i_{3}} A^{a_{3} i_{4}} \cdots A^{\left.a_{n-2} i_{n-1}\right]}\right) \eta^{a_{n-1}} \eta^{a_{n}}, \\
& \stackrel{(0)}{\bar{\omega}_{1}}=\epsilon_{0 i_{1} \ldots i_{n-1}} N_{a a_{1} \ldots a_{n-1}} A^{a_{1} i_{1}} \cdots A^{a_{n-1} i_{n-1}} \eta^{a},
\end{aligned}
$$

where we reverted to the one-form notation.

In consequence, so far we have computed the first-order deformation of the BRST charge that breaks the PT invariance like

$$
\bar{\omega}_{1}=\sum_{k=0}^{n-1} \stackrel{(k)}{\omega}_{1},
$$

such that the full first-order deformation of the BRST charge is

$$
\hat{\Omega}_{1}=\int d^{n-1} x\left(\omega_{1}+\bar{\omega}_{1}\right),
$$

with $\omega_{1}$ given in the Appendix $\mathrm{A}$. We emphasize that the solutions $\stackrel{(k)}{\omega}_{1}$ obtained in the above also include, for all $k<n-1$, the solutions corresponding to the associated 'homogeneous' equations $(k)$

$\gamma \bar{\omega}^{\prime}{ }_{1}=0$. In order to simplify the exposition we avoided the discussion regarding the selection procedure of these solutions such as to comply with obtaining some consistent components of the first-order deformation of the BRST charge at each value of the antighost number. It is however interesting to note that this procedure allows no new functions of the scalar fields beside $N_{a_{1} \ldots a_{n}}$ to parametrize the solution $\bar{\omega}_{1}$.

\subsection{Higher-order deformations}

Our next concern is to analyze the existence of higher-order deformations of the BRST charge. In view of this, we make the notations $\hat{\Omega}_{2}=\int d^{n-1} x b$ and $\left[\hat{\Omega}_{1}, \hat{\Omega}_{1}\right]=\int d^{n-1} x \Delta$, and observe that the equation (15), which governs the second-order deformation of the BRST charge, takes the local form

$$
\Delta=-2 s b+\partial_{i} m^{i}
$$


We mention that at this stage the entire BRST charge (84) must be taken into account, and not only the PT-breaking component $\bar{\omega}_{1}$. In view of the results from the previous subsection, combined with those from the Appendix $\mathrm{A}$, direct computation finally leads to

$$
\begin{aligned}
\Delta= & K^{a b c} t_{a b c}+\sum_{k=1}^{n-1} K_{a_{1} a_{2} \ldots a_{k}}^{a b c} \frac{\partial^{k} t_{a b c}}{\partial \varphi_{a_{1}} \partial \varphi_{a_{2}} \cdots \partial \varphi_{a_{k}}} \\
& +K_{d}^{a b c} t_{a b c}^{d}+\sum_{k=1}^{n-1} K_{d, a_{1} a_{2} \ldots a_{k}}^{a b c} \frac{\partial^{k} t_{a b c}^{d}}{\partial \varphi_{a_{1}} \partial \varphi_{a_{2}} \cdots \partial \varphi_{a_{k}}} \\
& +K^{a_{1} \ldots a_{n+1}} t_{a_{1} \ldots a_{n+1}}+\sum_{k=1}^{n-1} K_{b_{1} \ldots b_{k}}^{a_{1} \ldots a_{n+1}} \frac{\partial^{k} t_{a_{1} \ldots a_{n+1}}}{\partial \varphi_{b_{1}} \partial \varphi_{b_{2}} \cdots \partial \varphi_{b_{k}}}
\end{aligned}
$$

where

$$
\begin{aligned}
t_{a b c} & =W_{e c} M_{a b}^{c}+W_{e a} \frac{\partial W_{b c}}{\partial \varphi_{e}}+W_{e b} \frac{\partial W_{c a}}{\partial \varphi_{e}}, \\
t_{a b c}^{d} & =W_{e[a} \frac{\partial M_{b c]}^{d}}{\partial \varphi_{e}}+M_{e[a}^{d} M_{b c]}^{e}, \\
t_{a_{1} \ldots a_{n+1}} & =\frac{\partial N_{\left[a_{1} \ldots a_{n}\right.}}{\partial \varphi_{b}} W_{\left.a_{n+1}\right] b}+M_{\left[a_{1} a_{2}\right.}^{b} N_{\left.a_{3} \ldots a_{n+1}\right] b} .
\end{aligned}
$$

All the objects $K^{a b c}, K_{d}^{a b c}, K_{a_{1} a_{2} \ldots a_{k}}^{a b c}, K_{d, a_{1} a_{2} \ldots a_{k}}^{a b c}, K^{a_{1} \ldots a_{n}}$, and $K_{b_{1} \ldots b_{k}}^{a_{1} \ldots a_{n}}$ are polynomials that involve only undifferentiated ghosts, antighosts, and fields $B_{a}^{0 i}$ and $A_{i}^{a}$, none of them being BRST-exact. On the other hand, the equation (85) requires that $\Delta$ is $s$-exact modulo $\tilde{d}$, and, in fact, since $\Delta$ contains no derivatives, it demands that $\Delta$ must be $s$-exact. Since neither of the terms in (86) is so, it results that the consistency of the first-order deformation of the BRST charge asks that $\Delta$ must vanish. This takes place if and only if the equations

$$
\begin{aligned}
t_{a b c} & =0, \quad t_{a b c}^{d}=0 \\
t_{a_{1} \ldots a_{n+1}} & =0
\end{aligned}
$$

are simultaneously satisfied. As it has been shown in [8, the general solution to the equations (90) is of the form

$$
M_{a b}^{c}=\frac{\partial W_{a b}}{\partial \varphi_{c}}
$$

where $W_{a b}$ is an antisymmetric 'two-tensor' in the undifferentiated scalar fields, subject to the identities

$$
W_{e[a} \frac{\partial W_{b c]}}{\partial \varphi_{c}}=0 .
$$

Due to the fact that the antisymmetric functions $W_{a b}$ depend only on the undifferentiated scalar fields from the collection and verify the identities (93), they can be interpreted as the components of the Poisson two-tensor of a Poisson manifold with the target space locally parametrized by the scalar fields. Under these circumstances, the general solution of (911) can be represented like

$$
N_{a_{1} \ldots a_{n}}=f_{b\left[a_{1} \ldots a_{n-2}\right.} \frac{\partial W_{\left.a_{n-1} a_{n}\right]}}{\partial \varphi_{b}},
$$

where $f_{a_{1} \ldots a_{n}}$ are some completely antisymmetric constants. Consequently, we can take the secondorder deformation of the BRST charge to vanish, $\hat{\Omega}_{2}=0$, and, in fact, all the higher-order deformation equations are satisfied with the choice

$$
\hat{\Omega}_{k}=0 \text { for all } k \geq 2 .
$$


In conclusion, the deformed BRST charge, consistent to all orders in the coupling constant, simply reduces to the sum between the free BRST charge and the first-order deformation

$$
\hat{\Omega}=\Omega_{0}+g \hat{\Omega}_{1}=\Omega_{0}+g \int d^{n-1} x \sum_{k=0}^{n-1}\left(\begin{array}{c}
(k) \\
\omega_{1}
\end{array}+\stackrel{(k)}{\bar{\omega}_{1}}\right)=\Omega_{0}+g\left(\Omega_{1}+\bar{\Omega}_{1}\right),
$$

$\stackrel{(k)}{\bar{\omega}_{1}}$ and $\stackrel{(k)}{\omega}$ are listed in (75), (77)-(79), (181)-(182) and respectively in (132), (135)-(139), with the observation that $M_{a b}^{c}$ and $N_{a_{1} \ldots a_{n}}$ must be replaced with (92) and (94), while $W_{a b}$ are assumed to verify the identities (93).

\section{Deformation of the BRST-invariant Hamiltonian}

We now turn our attention to the BRST-invariant Hamiltonian (40), whose deformation is stipulated by the equations (9)-(10), etc. We will prove that the deformed BRST-invariant Hamiltonian, just like the BRST charge, stops at order one in the coupling constant and, moreover, is trivial (BRST-exact) with respect to the fully deformed BRST symmetry, which confirms the preservation of the topological behavior also at the level of the interacting theory.

Initially, we approach the equation (9), associated with its first-order deformation. Inserting (411) in (9) and using (41) and the Jacobi identity with respect to the Dirac bracket, we find that (92) is in fact equivalent to the equation $\left[\hat{H}_{1}-\left[K_{0}, \hat{\Omega}_{1}\right], \Omega_{0}\right]=0$, which shows that $\hat{H}_{1}-\left[K_{0}, \hat{\Omega}_{1}\right]$ is nothing but a BRST observable of the free theory. As it has been argued in Section 3. all the BRST observables are in this case trivial (BRST-exact), or, in other words, they belong to the same equivalence class as the trivial observable zero. In consequence, we can take

$$
\hat{H}_{1}=\left[K_{0}, \hat{\Omega}_{1}\right]
$$

where the function $K_{0}$ is displayed in (42). Next, we split $\hat{H}_{1}$ like the first-order deformation of the BRST charge, as the sum between the PT-invariant component $H_{1}$ and the PT-breaking part $\bar{H}_{1}$

$$
\hat{H}_{1}=H_{1}+\bar{H}_{1} \text {. }
$$

Recalling the similar decomposition of the first-order deformation of the BRST charge, we consequently get that

$$
H_{1}+\bar{H}_{1}=\left[K_{0}, \Omega_{1}\right]+\left[K_{0}, \bar{\Omega}_{1}\right] .
$$

It has been shown in 8 , that

$$
H_{1}=\left[K_{0}, \Omega_{1}\right]
$$

where the non-integrated density of $H_{1}$ has the expression

$$
\begin{aligned}
h_{1}= & -W_{a b} H_{\mu}^{a} A^{b \mu}-\frac{1}{2} M_{a b}^{c} A_{\mu}^{a} A_{\nu}^{b} B_{c}^{\mu \nu} \\
& -M_{a b}^{c}\left(\frac{1}{2} B_{c}^{i j} \eta^{a} \mathcal{P}_{i j}^{b}+A_{0}^{a} \mathcal{P}_{i j}^{b} \eta_{c}^{i j}+A_{0}^{a} \eta^{b} \mathcal{P}_{c}\right) \\
& +\frac{\partial W_{a b}}{\partial \varphi_{c}} P_{c}^{i}\left(H_{i}^{a} \eta^{b}+C_{i}^{a} A_{0}^{b}\right) \\
& +\frac{\partial M_{a b}^{c}}{\partial \varphi_{d}} P_{d i}\left(\eta^{a} A_{j}^{b} B_{c}^{i j}-\eta^{a} A_{0}^{b} B_{c}^{0 i}+2 A_{0}^{a} A_{j}^{b} \eta_{c}^{i j}\right) \\
& +\frac{1}{4}\left(\frac{\partial M_{a b}^{c}}{\partial \varphi_{d}} P_{d i j}+\frac{\partial^{2} M_{a b}^{c}}{\partial \varphi_{d} \partial \varphi_{e}} P_{d i} P_{e j}\right) \eta^{a} \eta^{b} B_{c}^{i j} \\
& +\sum_{k=2}^{n-1} A_{0}^{a} \frac{\partial^{L} \stackrel{(k)}{\omega}}{\partial \eta^{a}},
\end{aligned}
$$


and the notation $\partial^{L} / \partial \eta^{a}$ for the left derivative with respect to $\eta^{a}$ was employed. In (101) and further, $M_{a b}^{c}$ takes the form (92). By means of the relations (99)-(100), one finds that $\bar{H}_{1}$ checks the equation

$$
\bar{H}_{1}=\left[K_{0}, \bar{\Omega}_{1}\right] .
$$

On behalf of $\bar{\Omega}_{1}$ given in (102), we determine that the non-integrated density of $\bar{H}_{1}$ reads as

$$
\begin{aligned}
\bar{h}_{1}= & \epsilon_{0 i_{1} \ldots i_{n-1}} N_{a_{1} \ldots a_{n}} A^{a_{1} 0} A^{a_{2} i_{2}} \cdots A^{a_{n} i_{n-1}} \\
& -\epsilon_{0 i_{1} \ldots i_{n-1}} \eta^{a_{1}}\left(N_{a_{1} \ldots a_{n}} A^{a_{2} 0} \mathcal{P}^{a_{3}\left[i_{1} i_{2}\right.} A^{a_{4} i_{3}} \cdots A^{\left.a_{n} i_{n-1}\right]}\right. \\
& \left.-\frac{\partial N_{a_{1} \ldots a_{n}}}{\partial \varphi_{b}} A^{a_{2} 0} P_{b}^{\left[i_{1}\right.} A^{a_{3} i_{2}} \cdots A^{\left.a_{n} i_{n-1}\right]}\right)+\sum_{k=2}^{n-1} A_{0}^{a} \frac{\partial^{L} \stackrel{(k)}{\omega}_{1}}{\partial \eta^{a}},
\end{aligned}
$$

where in (103) and in what follows $N_{a_{1} \ldots a_{n}}$ takes the form (94).

In the next step we investigate the second-order deformation of the BRST-invariant Hamiltonian, subject to the equation (10). We observe that the third term in the right-hand side of the equation (10) vanishes since $\hat{\Omega}_{2}=0$. Making use of (97) and employing once more the Jacobi identity with respect to the Dirac bracket, it is easy to see that the second term in the right-hand side of (10) can be written like

$$
\left[\hat{H}_{1}, \hat{\Omega}_{1}\right]=\frac{1}{2}\left[K_{0},\left[\hat{\Omega}_{1}, \hat{\Omega}_{1}\right]\right]
$$

and it vanishes according to the fact (established in the previous section) that $\left[\hat{\Omega}_{1}, \hat{\Omega}_{1}\right]=0$. Then, we can set

$$
\hat{H}_{2}=0,
$$

which leads to the fact that the remaining higher-order equations are satisfied for

$$
\hat{H}_{k}=0, \quad k>2 .
$$

As a consequence, we can write the fully deformed BRST-invariant Hamiltonian like

$$
H_{\mathrm{B}}=H_{0 \mathrm{~B}}+g\left(H_{1}+\bar{H}_{1}\right)=H_{0 \mathrm{~B}}+g \hat{H}_{1},
$$

but also, taking into account (41), (96), and (97)

$$
H_{\mathrm{B}}=\left[K_{0}, \hat{\Omega}\right] \text {. }
$$

The last formula confirms the topological behavior of the interacting model. It stresses that $H_{\mathrm{B}}$ is not only invariant with respect to the deformed hamiltonian BRST symmetry, but also exact. This ends the deformation procedure of the BRST-invariant Hamiltonian for the free theory under study.

\section{Description of the interacting model}

With the deformed BRST charge and BRST-invariant Hamiltonian at hand, in the sequel we will be able to identify the main ingredients of the hamiltonian formulation for the resulting interacting model and, on these grounds, also the associated coupled lagrangian action and its gauge transformations. We recall that the deformed BRST charge is given in (96), with the corresponding components listed in (75), (77)-(79), (81)-(82) and respectively in (132), (135)-(139), while the deformed BRST-invariant Hamiltonian reads as in (107), with the non-integrated densities of $H_{1}$ 
and $\bar{H}_{1}$ given in (101) and (103). The functions $M_{a b}^{c}$ and $N_{a_{1} \ldots a_{n}}$ must be replaced with (92) and (94), while $W_{a b}$ are assumed to verify the identities (93).

It is well known that from the BRST charge and the BRST-invariant Hamiltonian one can withdraw the entire hamiltonian formulation of a gauge theory. Thus, from the terms of antighost number zero from (96) we see that only the secondary first-class constraints of the interacting model are deformed like

$$
\begin{gathered}
\tilde{G}_{a}^{(2)} \equiv-\left(D_{i}\right)_{a}{ }^{b} B_{b}^{0 i}+g W_{a b} H_{0}^{b}+g \epsilon_{0 i_{1} \ldots i_{n-1}} \times \\
\times N_{a a_{1} \ldots a_{n-1}} A^{a_{1} i_{1}} A^{a_{2} i_{2}} \cdots A^{a_{n-1} i_{n-1}} \approx 0, \\
\bar{G}_{i j}^{(2) a} \equiv-\bar{F}_{i j}^{a} \approx 0 \\
\bar{\gamma}_{a}^{(2) i} \equiv D^{i} \varphi_{a} \approx 0,
\end{gathered}
$$

while the primary ones are not affected by the deformation procedure, being given by (13)-(15). In the above we used the notations

$$
\begin{aligned}
\left(D_{i}\right)_{a}{ }^{b} & =\delta_{a}^{b} \partial_{i}+g \frac{\partial W_{a c}}{\partial \varphi_{b}} A_{i}^{c} \\
\bar{F}_{i j}^{a} & =\partial_{[i} A_{j]}^{a}+g \frac{\partial W_{b c}}{\partial \varphi_{a}} A_{i}^{b} A_{j}^{c}, \\
D^{i} \varphi_{a} & =\partial^{i} \varphi_{a}+g W_{a b} A^{b i} .
\end{aligned}
$$

The pieces of antighost number one in (96) reveal that only the Dirac brackets between the secondary first-class constraints are modified as

$$
\begin{gathered}
{\left[\tilde{G}_{a}^{(2)}, \tilde{G}_{b}^{(2)}\right]=-g\left(\frac{\partial W_{a b}}{\partial \varphi_{c}} \tilde{G}_{c}^{(2)}-\frac{\partial^{2} W_{a b}}{\partial \varphi_{c} \partial \varphi_{d}} B_{d 0 i} \bar{\gamma}_{c}^{(2) i}\right)} \\
+g \epsilon_{0 i_{1} \ldots i_{n-1}}\left(\frac{\partial N_{a b c_{1} \ldots c_{n-2}}}{\partial \varphi_{d}} \bar{\gamma}_{d}^{(2)\left[i_{1}\right.} A^{c_{1} i_{2}} \cdots A^{\left.c_{n-2} i_{n-1}\right]}\right. \\
\left.-N_{a b c_{1} \ldots c_{n-2}} \bar{G}^{(2) c_{1}\left[i_{1} i_{2}\right.} A^{c_{2} i_{3}} \cdots A^{\left.c_{n-2} i_{n-1}\right]}\right) \\
{\left[\tilde{G}_{a}^{(2)}, \bar{G}_{i j}^{(2) b}\right]=g\left(\frac{\partial W_{a c}}{\partial \varphi_{b}} \bar{G}_{i j}^{(2) c}-\frac{\partial^{2} W_{a c}}{\partial \varphi_{b} \partial \varphi_{d}} \bar{\gamma}_{d[i}^{(2)} A_{j]}^{c}\right)} \\
{\left[\tilde{G}_{a}^{(2)}, \bar{\gamma}_{b}^{(2) i}\right]=-g \frac{\partial W_{a b}}{\partial \varphi_{c}} \bar{\gamma}_{c}^{(2) i} .}
\end{gathered}
$$

If we compare the expressions (109)-(117) with the similar results from [8], we observe that here appear some supplementary contributions, due to the presence of the terms from the deformed BRST charge that break the PT invariance. Actually, from (96) one can read the entire tensor gauge structure of the first-class constraints by analyzing the various polynomials in ghosts and antighosts. For instance, the relations (115)-(117) signify that the gauge algebra of the first-class constraints is open (only closes on the first-class constraint surface) and, meanwhile, offer us the concrete form of the first-order structure functions. Still, higher-order structure functions appear by taking repeatedly the Dirac brackets among more than two deformed first-class constraint functions and can be read from the corresponding polynomials of higher antighost number in (96). Apart from exhibiting an intricate gauge algebra, the deformed first-class constraints remain reducible of order $(D-2)$, like those corresponding to the free model. The structure of the reducibility functions and relations is completely revealed by some of the terms of antighost number greater or equal to one from (96). These pieces are not modified by the presence of the terms that break the 
PT invariance, being the same like in [8]. However, for the sake of completeness, they are discussed in the Appendix B

Let us analyze now the deformed BRST-invariant Hamiltonian (107). Its component of antighost number zero,

$$
\hat{H}_{0}=\int d^{n-1} x\left(-H_{i}^{a} \bar{\gamma}_{a}^{(2) i}+\frac{1}{2} B_{a}^{i j} \bar{G}_{i j}^{(2) a}+A_{0}^{a} \tilde{G}_{a}^{(2)}\right),
$$

is nothing but the first-class Hamiltonian of the coupled model. From the terms of antighost number zero we determine the deformed Dirac brackets between the new first-class constraints and the first-class Hamiltonian of the interacting theory under the form

$$
\begin{gathered}
{\left[\hat{H}_{0}, G_{a}^{(1)}\right]=\tilde{G}_{a}^{(2)}} \\
{\left[\hat{H}_{0}, \tilde{G}_{a}^{(2)}\right]=g \frac{\partial W_{a b}}{\partial \varphi_{c}}\left(A_{0}^{b} \tilde{G}_{c}^{(2)}-H_{i}^{b} \bar{\gamma}_{c}^{(2) i}-\frac{1}{2} \bar{G}_{i j}^{(2) b} B_{c}^{i j}\right)} \\
+g \frac{\partial^{2} W_{a c}}{\partial \varphi_{b} \partial \varphi_{d}}\left(\frac{1}{2} B_{b}^{i j} \bar{\gamma}_{d[i}^{(2)} A_{j]}^{c}-B_{d 0 i} A_{0}^{c} \bar{\gamma}_{b}^{(2) i}\right) \\
-g \epsilon_{0 i_{1} \ldots i_{n-1}}\left(N_{a b_{1} \ldots b_{n-1}} A^{b_{1} 0} \bar{G}^{(2) b_{2}\left[i_{1} i_{2}\right.} A^{b_{3} i_{3}} \cdots A^{\left.b_{n-1} i_{n-1}\right]}\right] \\
-\frac{\left.\partial N_{a b_{1} \ldots b_{n-1}} A^{b_{1} 0} \bar{\gamma}_{c}^{(2)\left[i_{1}\right.} A^{b_{2} i_{2}} \ldots A^{\left.b_{n-1} i_{n-1}\right]}\right)}{\partial \varphi_{c}} \\
{\left[\hat{H}_{0}, G_{i j}^{(1) a}\right]=\bar{G}_{i j}^{(2) a},} \\
{\left[H_{T}, \gamma_{a}^{(1) i}\right]=\bar{\gamma}_{a}^{(2) i},} \\
{\left[\hat{H}_{0}, \bar{G}_{i j}^{(2) a}\right]=g\left(\frac{\partial W_{b c}}{\partial \varphi_{a}} A_{0}^{b} \bar{G}_{i j}^{(2) c}-\frac{\partial^{2} W_{c d}}{\partial \varphi_{a} \partial \varphi_{b}} A_{0}^{c} \bar{\gamma}_{b[i}^{(2)} A_{j]}^{d}\right)} \\
{\left[\hat{H}_{0}, \bar{\gamma}_{a}^{(2) i}\right]=g \frac{\partial W_{a b}}{\partial \varphi_{c}} A_{0}^{b} \bar{\gamma}_{c}^{(2) i} .}
\end{gathered}
$$

It is simple to see that the relations (118)-(124) also contain nontrivial contributions due to the terms from the deformed BRST-invariant Hamiltonian that break the PT invariance. The other terms in (107) reveal a new set of hamiltonian structure functions, that follow by taking repeatedly the Dirac brackets involving the interacting first-class Hamiltonian and more than two deformed first-class constraint functions. This sort of structure functions, as well as the equations satisfied by them, can be written down directly from (107) by identifying the suitable polynomials in ghosts and antighosts.

If we pass to the lagrangian formulation of the interacting theory (via the extended and total actions, together with the accompanying gauge symmetries), then we obtain that the interacting model is described by the lagrangian action

$$
\begin{aligned}
S\left[A_{\mu}^{a}, H_{\mu}^{a}, \varphi_{a}, B_{a}^{\mu \nu}\right]= & \int d^{n} x\left(H_{\mu}^{a} D^{\mu} \varphi_{a}+\frac{1}{2} B_{a}^{\mu \nu} \bar{F}_{\mu \nu}^{a}\right. \\
& \left.-\frac{1}{n} g \epsilon_{\mu_{1} \ldots \mu_{n}} N_{a_{1} \ldots a_{n}} A^{a_{1} \mu_{1}} \cdots A^{a_{n} \mu_{n}}\right),
\end{aligned}
$$

invariant under the gauge transformations

$$
\begin{gathered}
\delta_{\epsilon} A_{\mu}^{a}=\left(D_{\mu}\right)^{a}{ }_{b} \epsilon^{b}, \\
\delta_{\epsilon} \varphi_{a}=-g W_{a b} \epsilon^{b},
\end{gathered}
$$




$$
\begin{aligned}
\delta_{\epsilon} H_{\mu}^{a}= & \left(D^{\nu}\right)^{a}{ }_{b} \epsilon_{\mu \nu}^{b}-g \frac{\partial W_{b c}}{\partial \varphi_{a}} \epsilon^{b} H_{\mu}^{c} \\
& +g \frac{\partial^{2} W_{c d}}{\partial \varphi_{a} \partial \varphi_{b}}\left(\frac{1}{2} A^{c \nu} A^{d \rho} \epsilon_{b \mu \nu \rho}+A^{d \nu} \epsilon^{c} B_{b \mu \nu}\right) \\
& -g \epsilon_{\mu \mu_{1} \ldots \mu_{n-1}} \frac{\partial N_{b c_{1} \ldots c_{n-1}}}{\partial \varphi_{a}} \epsilon^{b} A^{c_{1} \mu_{1}} \cdots A^{c_{n-1} \mu_{n-1}}, \\
\delta_{\epsilon} B_{a}^{\mu \nu}= & \left(D_{\rho}\right)_{a}^{b} \epsilon_{b}^{\mu \nu \rho}+g W_{a b} \epsilon^{b \mu \nu}-g \frac{\partial W_{a b}}{\partial \varphi_{c}} \epsilon^{b} B_{c}^{\mu \nu} \\
& +g(n-1) g^{\mu \mu_{1}} g^{\nu \mu_{2}} \epsilon_{\mu_{1} \ldots \mu_{n}} N_{a b c_{1} \ldots c_{n-2}} \epsilon^{b} A^{c_{1} \mu_{3}} \cdots A^{c_{n-2} \mu_{n}} .
\end{aligned}
$$

At this stage it is clear that the deformation of the lagrangian gauge transformations is a consequence of the deformation of the first-class constraints like in (109)-(111). The above gauge transformations are on-shell $(n-2)$-order reducible, i.e., the reducibility relations only hold on the stationary surface of the field equations for the coupled action (125), while the accompanying gauge algebra is open (the commutators among the deformed gauge transformations only close on-shell). It is interesting to notice that the lagrangian formulation of the interacting BF theory contains contributions that break the PT invariance.

\section{Conclusion}

In conclusion in this paper we have investigated the PT-symmetry breaking, consistent hamiltonian interactions in all $n \geq 4$ spacetime dimensions that can be added to an abelian BF model involving a set of scalar fields, two sorts of one-forms, and a system of two-forms by means of the hamiltonian deformation procedure based on local BRST cohomology. The results related to the deformation of the BRST charge can be synthesized by the fact that only the first-order deformation is nontrivial, while its consistency relies on the existence of a Poisson two-tensor on a target space parametrized by the scalar fields. The deformation of the BRST-invariant Hamiltonian stops also at order one in the coupling constant and proves to be exact with respect to the deformed BRST charge, which confirms the topological character of the resulting interacting model. Both deformed BRST ingredients contain terms that are not PT invariant. The associated coupled theory is an interacting, topological BF model exhibiting an open gauge algebra (the Dirac brackets among the deformed first-class constraint functions only close on the first-class surface) and on-shell reducibility (the reducibility relations take place on the first-class surface).

\section{Acknowledgment}

The authors are partially supported by the European Commission FP6 program MRTN-CT-2004005104 and by the grant A304/2004 with the Romanian National Council for Academic Scientific Research (C.N.C.S.I.S.) and the Romanian Ministry of Education and Research (M.E.C.).

\section{A Deformed BRST charge with PT invariance}

In this Appendix we briefly recall the structure of the component from the deformed BRST charge (58) that preserves the PT invariance, namely, $\omega_{1}$. Various details on the expression of $\omega_{1}$ and on the interpretation of its various terms can be found in [8]. As it has been mentioned in Section 4 , $\omega_{1}$ is subject to the equation

$$
s \omega_{1}=\partial_{i} j^{i}
$$


for some local $j^{i}$. Taking into account the general cohomological properties of the free BF model described by (11) and discussed in the subsection 4.1. we can state that the most general decomposition of $\omega_{1}$ along the antighost number can be taken to stop in antighost number $(n-1)$

$$
\omega_{1}=\sum_{k=1}^{n-1} \stackrel{(k)}{\omega}_{1} .
$$

Furthermore, the last component, $\stackrel{(n-1)}{\omega}{ }_{1}$, belongs to the space of pure ghost number equal to $n$ from the cohomology of the exterior longitudinal derivative, $\gamma \stackrel{(n-1)}{\omega}{ }_{1}=0$, and thus it is of the form (63), with the corresponding 'invariant polynomial' from $H_{n-1}^{\text {inv }}(\delta \mid \tilde{d})$, and hence of the type (69). Requiring in addition that $\stackrel{(n-1)}{\omega}{ }_{1}$ preserves the PT invariance, it follows that we can write

$$
\stackrel{(n-1)}{\omega}{ }_{1}=-W_{a b}^{i_{1} \ldots i_{n-1}} \eta^{a} C_{i_{1} \ldots i_{n-1}}^{b}-\frac{(-)^{n}}{2}\left(M_{a b}^{c}\right)^{i_{1} \ldots i_{n-1}} \eta^{a} \eta^{b} \eta_{c i_{1} \ldots i_{n-1}},
$$

where

$$
\begin{aligned}
W_{a b}^{i_{1} \ldots i_{n-1}}= & \frac{\partial W_{a b}}{\partial \varphi_{c}} P_{c}^{i_{1} \ldots i_{n-1}}+\sum_{p=2}^{n-1} \sum_{1 \leq j_{1} \leq j_{2} \leq \cdots \leq j_{p}<n-1} \frac{\partial^{p} W_{a b}}{\partial \varphi_{a_{1}} \partial \varphi_{a_{2}} \cdots \partial \varphi_{a_{p}}} \times \\
& \times P_{a_{1}}^{\left[i_{1} \ldots i_{j_{1}}\right.} P_{a_{2}}^{i_{j_{1}+1} \ldots i_{j_{1}+j_{2}}} \cdots P_{a_{p-1}}^{i_{j_{1}+\cdots+j_{p-2}+1} \ldots i_{j_{1}+\cdots+j_{p-1}}} P_{a_{p}}^{\left.i_{j_{1}+\cdots+j_{p-1}+1} \ldots i_{n-1}\right]}
\end{aligned}
$$

and

$$
\begin{aligned}
\left(M_{a b}^{c}\right)^{i_{1} \ldots i_{n-1}}= & \frac{\partial M_{a b}^{c}}{\partial \varphi_{d}} P_{d}^{i_{1} \ldots i_{n-1}}+\sum_{p=2}^{n-1} \sum_{1 \leq j_{1} \leq j_{2} \leq \cdots \leq j_{p}<n-1} \frac{\partial^{p} M_{a b}^{c}}{\partial \varphi_{a_{1}} \partial \varphi_{a_{2}} \cdots \partial \varphi_{a_{p}}} \times \\
& \times P_{a_{1}}^{\left[i_{1} \ldots i_{j_{1}}\right.} P_{a_{2}}^{i_{j_{1}+1} \ldots i_{j_{1}+j_{2}}} \cdots P_{a_{p-1}}^{i_{j_{1}+\cdots+j_{p-2}+1} \ldots i_{j_{1}+\cdots+j_{p-1}}} P_{a_{p}}^{\left.i_{j_{1}+\cdots+j_{p-1}+1} \ldots i_{n-1}\right]}
\end{aligned}
$$

with $W_{a b}$ and $M_{a b}^{c}=-M_{a b}^{c}$ depending only on the undifferentiated scalar fields. The term of antighost number $(n-2)$ from $\omega_{1}$ is subject to the equation $\delta \stackrel{(n-1)}{\omega}{ }_{1}+\gamma \stackrel{(n-2)}{\omega}{ }_{1}=\partial_{i} \stackrel{(n-2){ }^{i}}{m}$ and is consequently given by

$$
\begin{aligned}
{\stackrel{(n-2)}{\omega}{ }_{1}=}^{(} & -W_{a b}^{i_{1} \ldots i_{n-2}} \eta^{a} C_{i_{1} \ldots i_{n-2}}^{b}+\frac{(-)^{n}}{2}\left(M_{a b}^{c}\right)^{i_{1} \ldots i_{n-2}} \eta^{a} \eta^{b} \eta_{c i_{1} \ldots i_{n-2}} \\
& -C_{n-1}^{1} W_{a b}^{i_{1} \ldots i_{n-2}} A^{a i_{n-1}} C_{i_{1} \ldots i_{n-1}}^{b} \\
& -\sum_{k=3}^{n}(-)^{k} C_{n-1}^{k-1} W_{a b}^{i_{1} \ldots i_{n-k}} \mathcal{P}^{a i_{n-k+1} \ldots i_{n-1}} C_{i_{1} \ldots i_{n-1}}^{b} \\
& -(-)^{n} C_{n-1}^{1}\left(M_{a b}^{c}\right)^{i_{1} \ldots i_{n-2}} A^{a i_{n-1}} \eta^{b} \eta_{c i_{1} \ldots i_{n-1}} \\
& -(-)^{n} \sum_{k=3}^{n}(-)^{k} C_{n-1}^{k-1}\left(M_{a b}^{c}\right)^{i_{1} \ldots i_{n-k}} \mathcal{P}^{a i_{n-k+1} \ldots i_{n-1}} \eta^{b} \eta_{c i_{1} \ldots i_{n-1}}
\end{aligned}
$$

where $C_{n}^{k}$ denotes the number of combinations of $k$ objects drawn from $n$, while the elements $W_{a b}^{i_{1} \ldots i_{n-2}}$ and $\left(M_{a b}^{c}\right)^{i_{1} \ldots i_{n-2}}$ result from (133)-(134) where we make the replacement $n \rightarrow n-1$. The component of antighost number $(n-3)$ is solution to the equation $\delta \stackrel{(n-2)}{\omega}{ }_{1}+\gamma \stackrel{(n-3)}{\omega}{ }_{1}=\partial_{i} \stackrel{(n-3)^{i}}{m}$ and reads as

$$
\stackrel{(n-3)}{\omega}{ }_{1}=-W_{a b}^{i_{1} i_{2} \ldots i_{n-3}} \eta^{a} C_{i_{1} i_{2} \ldots i_{n-3}}^{b}
$$




$$
\begin{aligned}
& -\frac{(-)^{n}}{2}\left(M_{a b}^{c}\right)^{i_{1} i_{2} \ldots i_{n-3}} \eta^{a} \eta^{b} \eta_{c i_{1} i_{2} \ldots i_{n-3}} \\
& -C_{n-2}^{1} W_{a b}^{i_{1} i_{2} \ldots i_{n-3}} A^{a i_{n-2}} C_{i_{1} i_{2} \ldots i_{n-2}}^{b} \\
& +\sum_{k=4}^{n}(-)^{k} C_{n-2}^{k-2} W_{a b}^{i_{1} i_{2} \ldots i_{n-k}} \mathcal{P}^{a i_{n-k+1} \ldots i_{n-2}} C_{i_{1} i_{2} \ldots i_{n-2}}^{b} \\
& +(-)^{n} C_{n-2}^{1}\left(M_{a b}^{c}\right)^{i_{1} i_{2} \ldots i_{n-3}} A^{a i_{n-2}} \eta^{b} \eta_{c i_{1} i_{2} \ldots i_{n-2}} \\
& -(-)^{n} \sum_{k=4}^{n}(-)^{k} C_{n-2}^{k-2}\left(M_{a b}^{c}\right)^{i_{1} i_{2} \ldots i_{n-k}} \mathcal{P}^{a i_{n-k+1} \ldots i_{n-2}} \eta^{b} \eta_{c i_{1} i_{2} \ldots i_{n-2}} \\
& -(-)^{n} \sum_{p=2}^{\left[\frac{n-2}{2}\right]} \sum_{n-p-1} \sum_{q=p+1}(-)^{q} C_{n-1}^{p} C_{n-p-1}^{q}\left(M_{a b}^{c}\right)^{i_{1} \ldots i_{n-p-q-1}} \times \\
& \times \mathcal{P}^{a j_{1} \ldots j_{q}} \mathcal{P}^{b l_{1} \ldots l_{p}} \eta_{c i_{1} \ldots i_{n-p-q-1} j_{1} \ldots j_{q} l_{1} \ldots l_{p}} \\
& -\frac{(-)^{n}}{2} \sum_{k=2}^{\left[\frac{n-1}{2}\right]}(-)^{k} C_{n-1}^{k} C_{n-k-1}^{k}\left(M_{a b}^{c}\right)^{i_{1} \ldots i_{n-2 k-1}} \times \\
& \times \mathcal{P}^{a j_{1} \ldots j_{k}} \mathcal{P}^{b l_{1} \ldots l_{k}} \eta_{c i_{1} \ldots i_{n-2 k-1} j_{1} \ldots j_{k} l_{1} \ldots l_{k}} \\
& +(-)^{n} C_{n-1}^{2}\left(M_{a b}^{c}\right)^{i_{1} \ldots i_{n-3}} A^{a i_{n-2}} A^{b i_{n-1}} \eta_{c i_{1} i_{2} \ldots i_{n-1}},
\end{aligned}
$$

where $W_{a b}^{i_{1} i_{2} \ldots i_{n-3}}$ and $\left(M_{a b}^{c}\right)^{i_{1} i_{2} \ldots i_{n-3}}$ are obtained from (133)-(134) via the shift $n \rightarrow n-2$. Along the same line, we deduce that the pieces of antighost number $(n-m)$, for $m=\overline{4, n-2}$, are given by

$$
\begin{aligned}
& \stackrel{(n-m)}{\omega}_{1}=-W_{a b}^{i_{1} i_{2} \ldots i_{n-m}} \eta^{a} C_{i_{1} i_{2} \ldots i_{n-m}}^{b} \\
& -C_{n-m+1}^{1} W_{a b}^{i_{1} i_{2} \ldots i_{n-m}} A^{a i_{n-m+1}} C_{i_{1} i_{2} \ldots i_{n-m+1}}^{b} \\
& -\sum_{k=m+1}^{n}(-)^{k+m} C_{n-m+1}^{k-m+1} W_{a b}^{i_{1} i_{2} \ldots i_{n-k}} \mathcal{P}^{a i_{n-k+1} \ldots i_{n-m+1}} C_{i_{1} i_{2} \ldots i_{n-m+1}}^{b} \\
& +\frac{(-)^{m+n}}{2}\left(M_{a b}^{c}\right)^{i_{1} \ldots i_{n-m}} \eta^{a} \eta^{b} \eta_{c i_{1} i_{2} \ldots i_{n-m}} \\
& -(-)^{m+n} C_{n-m+1}^{1}\left(M_{a b}^{c}\right)^{i_{1} \ldots i_{n-m}} A^{a i_{n-m+1}} \eta^{b} \eta_{c i_{1} i_{2} \ldots i_{n-m+1}} \\
& -\sum_{k=m+1}^{n}(-)^{k+n} C_{n-m+1}^{k-m+1}\left(M_{a b}^{c}\right)^{i_{1} \ldots i_{n-k}} \mathcal{P}^{a i_{n-k+1} \ldots i_{n-m+1}} \eta^{b} \eta_{c i_{1} i_{2} \ldots i_{n-m+1}} \\
& -(-)^{m+n} C_{n-m+2}^{2}\left(M_{a b}^{c}\right)^{i_{1} \ldots i_{n-m}} A^{a i_{n-m+1}} A^{b i_{n-m+2}} \eta_{c i_{1} i_{2} \ldots i_{n-m+2}} \\
& +\frac{1}{2} \sum_{k=2}^{\left[\frac{n-m+2}{2}\right]}(-)^{k+n+m} C_{n-m+2}^{k} C_{n-m-k+2}^{k}\left(M_{a b}^{c}\right)^{i_{1} \ldots i_{n-2 k-m+2}} \times \\
& \times \mathcal{P}^{a j_{1} \ldots j_{k}} \mathcal{P}^{b l_{1} \ldots l_{k}} \eta_{c i_{1} \ldots i_{n-2 k-m+2} j_{1} \ldots j_{k} l_{1} \ldots l_{k}} \\
& +\sum_{p=2}^{\left[\frac{n-m+1}{2}\right]} \sum_{q=p+1}^{n-m-p+2}(-)^{q+n+m} C_{n-m+2}^{p} C_{n-m-p+2}^{q}\left(M_{a b}^{c}\right)^{i_{1} \ldots i_{n-m-p-q+2}} \times \\
& \times \mathcal{P}^{a j_{1} \ldots j_{q}} \mathcal{P}^{b l_{1} \ldots l_{p}} \eta_{c i_{1} \ldots i_{n-m-p-q+2} j_{1} \ldots j_{q} l_{1} \ldots l_{p}} \\
& -\sum_{k=m+1}^{n}(-)^{k+n} C_{n-m+1}^{k-m+1} C_{n-m+2}^{1}\left(M_{a b}^{c}\right)^{i_{1} \ldots i_{n-k}} \times \\
& \times \mathcal{P}^{a i_{n-k+1} \ldots i_{n-m+1}} A^{b i_{n-m+2}} \eta_{c i_{1} i_{2} \ldots i_{n-m+2}},
\end{aligned}
$$


where the elements $W_{a b}^{i_{1} i_{2} \ldots i_{n-m}}$ and $\left(M_{a b}^{c}\right)^{i_{1} \ldots i_{n-m}}$ are deduced from (133)-(134) where we set $n \rightarrow n-m+1$. Finally, the components of antighost number one and zero from the first-order deformation $\omega_{1}$ respectively take the form

$$
\begin{aligned}
& \stackrel{(1)}{\omega}_{1}=-\frac{\partial W_{a b}}{\partial \varphi_{c}} P_{c}^{i}\left(\eta^{a} C_{i}^{b}+2 A^{a j} C_{i j}^{b}\right)+W_{a b} \mathcal{P}^{a i j} C_{i j}^{b} \\
&+\frac{\partial M_{a b}^{c}}{\partial \varphi_{d}} P_{d i}\left(\frac{1}{2} \eta^{a} \eta^{b} B_{c}^{0 i}+2 A_{j}^{a} \eta^{b} \eta_{c}^{i j}+3 A_{j}^{a} A_{k}^{b} \eta_{c}^{i j k}\right) \\
&-M_{a b}^{c}\left(\mathcal{P}_{i j}^{a} \eta^{b} \eta_{c}^{i j}+\mathcal{P}_{[i j}^{a} A_{k]}^{b} \eta_{c}^{i j k}+\frac{1}{2} \eta^{a} \eta^{b} \mathcal{P}_{c}\right), \\
& \stackrel{(0)}{\omega}_{1}=W_{a b}\left(\eta^{a} H_{0}^{b}-A^{a i} C_{i}^{b}\right)+M_{a b}^{c}\left(A_{i}^{a} \eta^{b} B_{c}^{0 i}-A_{i}^{a} A_{j}^{b} \eta_{c}^{i j}\right) .
\end{aligned}
$$

In conclusion, the first-order deformation of the BRST charge for the model under study that preserves the PT invariance is the sum among the components (132) and (135)-(139).

\section{B Reducibility of the deformed interacting model}

Here, we investigate the reducibility of the first-class constraints corresponding to the interacting model discussed in Section [6. In view of this, we analyze some of the terms contained in the deformed BRST charge given in (96), with the corresponding components listed in (75), (77) - (79), (81) - (82) and respectively in (132), (135)-(139). From the elements in (96) with antighost number one that are linear in the ghosts one reads the first-order reducibility relations

$$
\begin{aligned}
\left(\bar{Z}_{i_{1} i_{2} i_{3}}^{a}\right)_{b}^{i j} \bar{G}_{i j}^{(2) b}+\left(\bar{Z}_{i_{1} i_{2} i_{3}}^{a}\right)_{i}^{b} \bar{\gamma}_{b}^{(2) i}=0 \\
\left(\bar{Z}_{a}^{i_{1} i_{2}}\right)_{b}^{i j} \bar{G}_{i j}^{(2) b}+\left(\bar{Z}_{a}^{i_{1} i_{2}}\right)_{i}^{b} \bar{\gamma}_{b}^{(2) i}=0,
\end{aligned}
$$

and also the associated first-order reducibility functions

$$
\begin{aligned}
\left(\bar{Z}_{i_{1} i_{2} i_{3}}^{a}\right)_{b}^{i j} & =\frac{1}{2}\left(D_{\left[i_{1}\right.}\right)_{b}^{a} \delta_{i_{2}}^{i} \delta_{\left.i_{3}\right]}^{j}, \\
\left(\bar{Z}_{i_{1} i_{2} i_{3}}^{a}\right)_{i}^{b} & =g \frac{\partial^{2} W_{c d}}{\partial \varphi_{a} \partial \varphi_{b}} g_{i\left[i_{1}\right.} A_{i_{2}}^{c} A_{\left.i_{3}\right]}^{d}, \\
\left(\bar{Z}_{a}^{i_{1} i_{2}}\right)_{b}^{i j} & =-\frac{1}{2} g W_{a b}\left(g^{i_{1} i} g^{i_{2} j}-g^{i_{1} j} g^{i_{2} i}\right), \\
\left(\bar{Z}_{a}^{i_{1} i_{2}}\right)_{i}^{b} & =-\left(D^{\left[i_{1}\right.}\right)_{a}^{b} \delta_{i}^{\left.i_{2}\right]},
\end{aligned}
$$

where

$$
\left(D_{i}\right)^{a}{ }_{b}=\delta_{b}^{a} \partial_{i}-g \frac{\partial W_{b c}}{\partial \varphi_{a}} A_{i}^{c} .
$$

The part from (96) that is linear in the ghosts with the pure ghost number equal to $k+1 \geq 3$ contains polynomials of antighost number $k \geq 2$, which are at least quadratic in the antighosts, so the reducibility relations of order $k \geq 2$ only close on the first-class constraint surface (on-shell). For instance, in pure ghost number three $(k+1=3)$ the second-order reducibility relations take place on-shell

$$
\begin{aligned}
& \left(\bar{Z}_{i_{1} i_{2} i_{3} i_{4}}^{a}\right)_{b}^{j_{1} j_{2} j_{3}}\left(\bar{Z}_{j_{1} j_{2} j_{3}}^{b}\right)_{c}^{i j} f_{i j}^{c}+\left(\bar{Z}_{i_{1} i_{2} i_{3} i_{4}}^{a}\right)_{j_{1} j_{2}}^{b}\left(\bar{Z}_{b}^{j_{1} j_{2}}\right)_{c}^{i j} f_{i j}^{c} \\
& =-g\left(\frac{\partial W_{b c}}{\partial \varphi_{a}} \bar{G}_{\left[i_{1} i_{2}\right.}^{(2) b} f_{\left.i_{3} i_{4}\right]}^{c}-\frac{\partial^{2} W_{c d}}{\partial \varphi_{a} \partial \varphi_{b}} \bar{\gamma}_{b\left[i_{1}\right.}^{(2)} A_{i_{2}}^{c} f_{\left.i_{3} i_{4}\right]}^{d}\right),
\end{aligned}
$$




$$
\begin{aligned}
& \left(\bar{Z}_{a}^{i_{1} i_{2} i_{3}}\right)_{j_{1} j_{2}}^{b}\left(\bar{Z}_{b}^{j_{1} j_{2}}\right)_{i}^{c} f_{c}^{i}+\left(\bar{Z}_{a}^{i_{1} i_{2} i_{3}}\right)_{b}^{j_{1} j_{2} j_{3}}\left(\bar{Z}_{j_{1} j_{2} j_{3}}^{b}\right)_{i}^{c} f_{c}^{i} \\
& =g\left(\frac{\partial W_{a b}}{\partial \varphi_{c}} \bar{G}^{(2) b\left[i_{1} i_{2}\right.} f_{c}^{\left.i_{3}\right]}-\frac{\partial^{2} W_{a b}}{\partial \varphi_{c} \partial \varphi_{d}} \bar{\gamma}_{c}^{(2)\left[i_{1}\right.} A^{b i_{2}} f_{d}^{\left.i_{3}\right]}\right)
\end{aligned}
$$

where $f_{c}^{i}$ and $f_{i j}^{c}$ are some arbitrary, smooth functions (the latter ones are antisymmetric in their spatial indices). The reducibility functions involved in (147) and (148) take the form

$$
\begin{aligned}
\left(\bar{Z}_{i_{1} i_{2} i_{3} i_{4}}^{a}\right)_{b}^{j_{1} j_{2} j_{3}} & =-\frac{1}{3 !}\left(D_{\left[i_{1}\right.}\right)_{b}^{a} \delta_{i_{2}}^{j_{1}} \delta_{i_{3}}^{j_{2}} \delta_{\left.i_{4}\right]}^{j_{3}}, \\
\left(\bar{Z}_{i_{1} i_{2} i_{3} i_{4}}^{a}\right)_{j_{1} j_{2}}^{b} & =-\frac{g}{2} g_{j_{1} k_{1}} g_{j_{2} k_{2}} \frac{\partial^{2} W_{c d}}{\partial \varphi_{a} \partial \varphi_{b}} \delta_{\left[i_{1}\right.}^{k_{1}} \delta_{i_{2}}^{k_{2}} A_{i_{3}}^{c} A_{\left.i_{4}\right]}^{d}, \\
\left(\bar{Z}_{a}^{i_{1} i_{2} i_{3}}\right)_{j_{1} j_{2}}^{b} & =\frac{1}{2}\left(D^{\left[i_{1}\right.}\right)_{a}^{b} \delta_{j_{1}}^{i_{2}} \delta_{j_{2}}^{\left.i_{3}\right]}, \\
\left(\bar{Z}_{a}^{i_{1} i_{2} i_{3}}\right)_{b}^{j_{1} j_{2} j_{3}} & =\frac{g}{3 !} W_{a b} \sum_{\sigma \in S_{3}}(-)^{\sigma} g^{i_{1} j_{\sigma(1)}} g^{i_{2} j_{\sigma(2)}} g^{i_{3} j_{\sigma(3)}} .
\end{aligned}
$$

In (152) $S_{3}$ is the set of permutations of the elements $\{1,2,3\}$ and $(-)^{\sigma}$ denotes the parity of the permutation $\sigma$ from $S_{3}$.

Reprising a similar analysis with respect to the terms from (96) linear in the ghosts with the pure ghost number equal to $(p+1), p=\overline{3, n-3}$, we deduce some reducibility relations of order $p$ that also close on-shell, namely,

$$
\begin{aligned}
& \left(\bar{Z}_{i_{1} \ldots i_{p+2}}^{a}\right)_{b}^{j_{1} \ldots j_{p+1}}\left(\bar{Z}_{j_{1} \ldots j_{p+1}}^{b}\right)_{c}^{k_{1} \ldots k_{p}}+\left(\bar{Z}_{i_{1} \ldots i_{p+2}}^{a}\right)_{j_{1} \ldots j_{p}}^{b}\left(\bar{Z}_{b}^{j_{1} \ldots j_{p}}\right)_{c}^{k_{1} \ldots k_{p}} \approx 0, \\
& \left(\bar{Z}_{a}^{i_{1} \ldots i_{p+1}}\right)_{j_{1} \ldots j_{p}}^{b}\left(\bar{Z}_{b}^{j_{1} \ldots j_{p}}\right)_{k_{1} \ldots k_{p-1}}^{c}+\left(\bar{Z}_{a}^{i_{1} \ldots i_{p+1}}\right)_{b}^{j_{1} \ldots j_{p+1}}\left(\bar{Z}_{j_{1} \ldots j_{p+1}}^{b}\right)_{k_{1} \ldots k_{p-1}}^{c} \approx 0 \text {, }
\end{aligned}
$$

with the reducibility functions

$$
\begin{aligned}
\left(\bar{Z}_{i_{1} \ldots i_{p+2}}^{a}\right)_{b}^{j_{1} \ldots j_{p+1}} & =\frac{(-)^{p+1}}{(p+1) !}\left(D_{\left[i_{1}\right.}\right)_{b}^{a} \delta_{i_{2}}^{j_{1}} \cdots \delta_{\left.i_{p+2}\right]}^{j_{p+1}}, \\
\left(\bar{Z}_{i_{1} \ldots i_{p+2}}^{a}\right)_{j_{1} \ldots j_{p}}^{b} & =-\frac{(-)^{p} g}{p !} g_{j_{1} k_{1}} \cdots g_{j_{p} k_{p}} \frac{\partial^{2} W_{c d}}{\partial \varphi_{a} \partial \varphi_{b}} \delta_{\left[i_{1}\right.}^{k_{1}} \cdots \delta_{i_{p}}^{k_{p}} A_{i_{p+1}}^{c} A_{\left.i_{p+2}\right]}^{d}, \\
\left(\bar{Z}_{a}^{i_{1} \ldots i_{p+1}}\right)_{j_{1} \ldots j_{p}}^{b} & =\frac{(-)^{p}}{p !}\left(D^{\left[i_{1}\right.}\right)_{a}^{b} \delta_{j_{1}}^{i_{2}} \cdots \delta_{j_{p}}^{\left.i_{p+1}\right]}, \\
\left(\bar{Z}_{a}^{i_{1} \ldots i_{p+1}}\right)_{b}^{j_{1} \ldots j_{p+1}} & =\frac{(-)^{p} g}{(p+1) !} W_{a b} \sum_{\sigma \in S_{p+1}}(-)^{\sigma} g^{i_{1} j_{\sigma(1)}} g^{i_{2} j_{\sigma(2)}} \cdots g^{i_{p+1} j_{\sigma(p+1)}}
\end{aligned}
$$

In (158) $S_{p+1}$ is the set of permutations of the elements $\{1, \ldots, p+1\}$, while $(-)^{\sigma}$ stands for the parity of the permutation $\sigma$ belonging to $S_{p+1}$. The reducibility relations of maximum order, $(n-2)$, follow from the elements in (96) that are linear in the ghosts with the pure ghost number equal to $(n-1)$

$$
\begin{aligned}
& \left(\bar{Z}_{a}^{i_{1} \ldots i_{n-1}}\right)_{j_{1} \ldots j_{n-2}}^{b}\left(\bar{Z}_{b}^{j_{1} \ldots j_{n-2}}\right)_{k_{1} \ldots k_{n-3}}^{c} f_{c}^{k_{1} \ldots k_{n-3}} \\
+ & \left(\bar{Z}_{a}^{i_{1} \ldots i_{n-1}}\right)_{b}^{j_{1} \ldots j_{n-1}}\left(\bar{Z}_{j_{1} \ldots j_{n-1}}^{b}\right)_{k_{1} \ldots k_{n-3}}^{c} f_{c}^{k_{1} \ldots k_{n-3}} \\
= & -g\left(\frac{\partial^{2} W_{a b}}{\partial \varphi_{c} \partial \varphi_{d}} \bar{\gamma}_{c}^{(2)\left[i_{1}\right.} A^{b i_{2}} f_{d}^{\left.i_{3} \ldots i_{n-1}\right]}-\frac{\partial W_{a b}}{\partial \varphi_{c}} \bar{G}^{(2) b\left[i_{1} i_{2}\right.} f_{c}^{\left.i_{3} \ldots i_{n-1}\right]}\right),
\end{aligned}
$$




$$
\begin{aligned}
& \left(\bar{Z}_{a}^{i_{1} \ldots i_{n-1}}\right)_{j_{1} \ldots j_{n-2}}^{b}\left(\bar{Z}_{b}^{j_{1} \ldots j_{n-2}}\right)_{c}^{k_{1} \ldots k_{n-2}} f_{k_{1} \ldots k_{n-2}}^{c} \\
& +\left(\bar{Z}_{a}^{i_{1} \ldots i_{n-1}}\right)_{b}^{j_{1} \ldots j_{n-1}}\left(\bar{Z}_{j_{1} \ldots j_{n-1}}^{b}\right)_{c}^{k_{1} \ldots k_{n-2}} f_{k_{1} \ldots k_{n-2}}^{c} \\
= & -g \frac{\partial W_{a b}}{\partial \varphi_{c}} \bar{\gamma}_{c}^{(2)\left[i_{1}\right.} f^{\left.b i_{2} \ldots i_{n-1}\right]}
\end{aligned}
$$

with $f_{c}^{k_{1} \ldots k_{n-3}}$ and $f_{k_{1} \ldots k_{n-2}}^{c}$ some arbitrary, smooth functions, completely antisymmetric in their spatial indices. The reducibility functions of order $(n-2)$ are consequently given by

$$
\begin{aligned}
\left(\bar{Z}_{a}^{i_{1} \ldots i_{n-1}}\right)_{j_{1} \ldots j_{n-2}}^{b} & =\frac{(-)^{n}}{(n-2) !}\left(D^{\left[i_{1}\right.}\right)_{a}^{b} \delta_{j_{1}}^{i_{2}} \cdots \delta_{j_{n-2}}^{\left.i_{n-1}\right]}, \\
\left(\bar{Z}_{a}^{i_{1} \ldots i_{n-1}}\right)_{b}^{j_{1} \ldots j_{n-1}} & =\frac{(-)^{n} g}{(n-1) !} W_{a b} \sum_{\sigma \in S_{n-1}}(-)^{\sigma} g^{i_{1} j_{\sigma(1)}} g^{i_{2} j_{\sigma(2)}} \cdots g^{i_{n-1} j_{\sigma(n-1)}} .
\end{aligned}
$$

The notations $S_{n-1}$ and $(-)^{\sigma}$ have the same meanings like before.

\section{References}

[1] M. Henneaux and C. Teitelboim, Quantization of Gauge Systems, Princeton University Press, Princeton 1992

[2] E. S. Fradkin and G. A. Vilkovisky, Phys. Lett. B55 (1975) 224; I. A. Batalin and G. A. Vilkovisky, Phys. Lett. B69 (1977) 309; E. S. Fradkin and T. E. Fradkina, Phys. Lett. B72 (1978) 343; I. A. Batalin and E. S. Fradkin, Phys. Lett. B122 (1983) 157; M. Henneaux, Phys. Rept. 126 (1985) 1

[3] R. Ferraro, M. Henneaux and M. Puchin, J. Math. Phys. 34 (1993) 2757, hep-th/9210070

[4] G. Barnich, Mod. Phys. Lett. A9 (1994) 665, hep-th/9310167

[5] G. Barnich and M. Henneaux, J. Math. Phys. 37 (1996) 5273, hep-th/9210070

[6] C. Bizdadea, Acta Phys. Polon. B32 (2001) 2843, hep-th/0003199

[7] C. Bizdadea, E. M. Cioroianu and S. O. Saliu, Class. Quantum Grav. 17 (2000) 2007, hep-th/0003192 C. Bizdadea, L. Saliu and S. O. Saliu, Int. J. Mod. Phys. A15 (2000) 893, hep-th/0003191, C. Bizdadea and S. O. Saliu, Phys. Scripta 62 (2000) 261

[8] C. Bizdadea, C. C. Ciobîrcă, E. M. Cioroianu, S. O. Saliu and S. C. Săraru, J. High Energy Phys. 0301 (2003) 049, hep-th/0302037

[9] C. Bizdadea, Mod. Phys. Lett. A15 (2000) 2047, hep-th/0201059

[10] C. Bizdadea, E. M. Cioroianu and S. O. Saliu, Int. J. Mod. Phys. A17 (2002) 2191, hep-th/0206186

[11] D. Birmingham, M. Blau, M. Rakowski and G. Thompson, Phys. Rept. 209 (1991) 129

[12] M. O. Katanaev and I. V. Volovich, Phys. Lett. B175 (1986) 413, hep-th/0209014 Annals Phys. (N.Y.) 197 (1990) 1; S. N. Solodukhin, Class. Quantum Grav. 10 (1993) 1011

[13] H.-J. Schmidt, J. Math. Phys. 32 (1991) 1562; N. Ikeda and K. I. Izawa, Prog. Theor. Phys. 90 (1993) 237, hep-th/9304012 D. Grumiller, W. Kummer and D. V. Vassilevich, Phys. Rept. 369 (2002) 327, hep-th/0204253 
[14] J. Brown, Lower Dimensional Gravity, World Scientific, Singapore 1988

[15] C. Teitelboim, Phys. Lett. B126 (1983) 41; R. Jackiw, Nucl. Phys. B252 (1985) 343

[16] N. Ikeda, Annals Phys. (N.Y.) 235 (1994) 435, hep-th/9312059, T. Strobl, Phys. Rev. D50 (1994) 7346, hep-th/9403121

[17] P. Schaller and T. Strobl, Mod. Phys. Lett. A9 (1994) 3129, hep-th/9405110

[18] A. Yu. Alekseev, P. Schaller and T. Strobl, Phys. Rev. D52 (1995) 7146, hep-th/9505012

[19] A. S. Cattaneo and G. Felder, Mod. Phys. Lett. A16 (2001) 179, hep-th/0102208 Commun. Math. Phys. 212 (2000) 591, math.QA/9902090

[20] T. Klösch and T. Strobl, Class. Quantum Grav. 13 (1996) 965, gr-qc/9508020; Erratum- Class. Quantum Grav. 14 (1997) 825; Class. Quantum Grav. 13 (1996) 2395, gr-qc/9511081; Class. Quantum Grav. 14 (1997) 1689, hep-th/9607226

[21] T. Strobl, Gravity in Two Spacetime Dimensions, Habilitation thesis RWTH Aachen, Aachen 1999, hep-th/0011240

[22] N. Ikeda, J. High Energy Phys. 0011 (2000) 009, hep-th/0010096 J. High Energy Phys. 0107 (2001) 037, hep-th/0105286

[23] G. Barnich, F. Brandt and M. Henneaux, Commun. Math. Phys. 174 (1995) 93, hep-th/9405194

[24] G. Barnich, F. Brandt and M. Henneaux, Commun. Math. Phys. 174 (1995) 57, hep-th/9405109 Phys. Rept. 338 (2000) 439, hep-th/0002245 